\title{
Analytical and Semianalytical Treatment of the Collinear Points in the Photogravitational Relativistic RTBP
}

\author{
S. E. Abd El-Bar ${ }^{1,2}$ and F. A. Abd El-Salam ${ }^{3,4}$ \\ ${ }^{1}$ Department of Applied Mathematics, Faculty of Applied Science, Taibah University, Al-Madinah, Saudi Arabia \\ ${ }^{2}$ Department of Mathematics, Faculty of Science, Tanta University, Tanta, Egypt \\ ${ }^{3}$ Department of Mathematics, Faculty of Science, Taibah University, Al-Madinah, Saudi Arabia \\ ${ }^{4}$ Department of Astronomy, Faculty of Science, Cairo University, Cairo 12613, Egypt
}

Correspondence should be addressed to S. E. Abd El-Bar; so_abdelbar@yahoo.com

Received 22 April 2013; Accepted 24 July 2013

Academic Editor: Alexander P. Seyranian

Copyright (C) 2013 S. E. Abd El-Bar and F. A. Abd El-Salam. This is an open access article distributed under the Creative Commons Attribution License, which permits unrestricted use, distribution, and reproduction in any medium, provided the original work is properly cited.

\begin{abstract}
The problem of restricted three bodies is considered. The treatment is carried out within the framework of the post-Newtonian approximation. The primaries also are assumed to be radiant sources. The locations of the collinear points are computed. Series forms of these locations are obtained as new results. A Mathematica program is constructed so as to draw the locations of collinear points versus the whole range of the mass ratio $\mu$ taking into account the photo-gravitational effects and/or the relativistic corrections. Analyses of these figures are addressed.
\end{abstract}

\section{Introduction}

The restricted three-body problem (in brief RTBP) is the most celebrated problem in celestial mechanics, wherein one is interested in the motion of a test particle, a body of negligible mass called infinitesimal mass in the presence of two massive bodies called primaries revolving around their common centre of mass. Attempts at its solution laid the foundation for dynamical systems theory and alerted Poincaré in the 1890s to the existence of deterministic chaos within Newtonian mechanics. There is no general analytical solution in terms of simple algebraic functions for the problem of three gravitating bodies of comparable masses. Except in a few very specific cases the problem has to be solved numerically. Are there any points where an infinitesimal mass could orbit around the centre of mass with the same period as the primaries? Euler found three such points, the collinear points, on the line joining the primaries. Lagrange found two additional points, the fourth and fifth triangular points. They are not collinear with $m_{1}$ and $m_{2}$ but are such that the three masses are at the corners of an equilateral triangle. The five points are called the equilibrium points.
These locations of the equilibrium points can be found by setting the first derivatives of the potential to zero. Note that, in the line joining the two masses, the equivalent potential at the collinear points is a maximum, and therefore these points are unstable; these points are actually saddle points. In several spacecrafts which are in orbit or are planned to be in orbit around the collinear points (e.g., SOHO at the interior collinear point and MAP at $L_{2}$ ), this instability has already used in consuming small expenditure of fuel which is presumably needed to keep them there.

The classical restricted three-body problem concerned with the determination of the locations of the libration points and investigating their linear/nonlinear stability. The RTBP becomes photo-gravitational when one or both of the masses of the primaries is an intense emitter of radiation, Kumar and Ishwar [1]. The restricted three body problem describes the dynamical system more accurately on account of realistic assumptions of the potential field and of the motion of the primaries, Narayan and Kumar [2].

The location and stability of the libration points in the problem of three bodies are subject to modifications when including one or more of additional forces in the potential 
of the classical problem as the radiation pressure component of the radiation drag which is the next most powerful component after the gravitational forces, Schuerman [3]; the oblateness of the primaries, Oberti and Vienne [4]; the primaries orbits being elliptic, Kumar and Ishwar [5]; the relativistic corrections to RTBP Abd El-Salam and Abd ElBar [6], Douskos and Perdios [7], and Ragos et al. [8]; the aerodynamic drag, Markakis et al. [9]; Douskos [10] and the eternal radiation pressure as the effect of the direct solar radiation pressure on the equilibrium points in the EarthMoon system, Poynting-Robertson (P-R) drag, and so forth.

The literature is wealthy of works on the subject of the restricted three body problem in classical form since its eminent pioneer Lagrange to the very recent date when considering different sources of perturbations and it will be worth noting to sketch some of these most important as well as recent works: Sharma [11], Hallan and Rana [12], Sándor and Érdi [13], Floría [14], Markeev [15], Palacián et al. [16, 17], Bruno and Varin [18], Ammar [19], Narayan et al. [20-22], Kushvah and [23-25], Douskos [26], Shankaran et al. [27].

In this work, we consider the collinear equilibrium points of the relativistic RTBP amended with the photo-gravitational effects of the two primaries. We determine new formulas for approximate positions of the collinear points as series expansions. Terms are ordered in $\mu$ and $1 / c^{2}$ under the considered perturbations.

The rest of the paper is organized as follows. In Section 2 we formulated the equations of motion of the infinitesimal mass in the relativistic RTBP in a synodic frame of reference taking into account the radiation of the primaries. In Section 3 we computed the perturbed locations of the collinear libration points. In Section 4 we designed a Mathematica program to draw the locations of collinear points $L_{i}, i=$ $1,2,3$, versus the whole range of the mass ratio $\mu$ taking into account the photogravitational effects and/or the relativistic corrections. In Section 5 we concluded our work and highlighted the differences between our work and previous works. Also we mentioned our forthcoming works for the sake of completeness.

\section{Equations of Motion}

The equations of motion of the infinitesimal mass in the relativistic RTBP in a synodic frame of reference $(\xi, \eta)$, in which the primary coordinates on the $\xi$-axis $(-\mu, 0),(1-\mu, 0)$ are kept fixed and the origin at the center of mass, are given by Brumberg [28] and Bhatnagar and Hallan [29]:

$$
\begin{aligned}
& \ddot{\xi}-2 n \dot{\eta}=\frac{\partial U}{\partial \xi}-\frac{d}{d t}\left(\frac{\partial U}{\partial \dot{\xi}}\right), \\
& \ddot{\eta}+2 n \dot{\xi}=\frac{\partial U}{\partial \eta}-\frac{d}{d t}\left(\frac{\partial U}{\partial \dot{\eta}}\right)
\end{aligned}
$$

with

$$
\begin{gathered}
n=1+\frac{1}{2 c^{2}}(\mu(1-\mu)-3), \\
r=\sqrt{\left(\xi^{2}+\eta^{2}\right)},
\end{gathered}
$$

where $U$ is the potential-like function of the relativistic RTBP, which can be written as composed of two components $U_{c+\mathrm{ph}}$ and $U_{r+\mathrm{ph}}$, namely, on respective the potential of the classical RTBP gravitational potential and the relativistic terms corrected for the radiation of the primaries which lead to mass reduction:

$$
U=U_{c+\mathrm{ph}}+U_{r+\mathrm{ph}}
$$

where $U_{c+\text { ph }}$ and $U_{r+\text { ph }}$ are given by

$$
\begin{aligned}
& U_{c+\mathrm{ph}}=\frac{r^{2}}{2}+\frac{q_{1}(1-\mu)}{r_{1}}+\frac{q_{2} \mu}{r_{2}}, \\
& U_{r+\mathrm{ph}}= \frac{r^{2}}{2 c^{2}}(\mu(1-\mu)-3)+\frac{1}{8 c^{2}} \\
& \times\left((\xi+\dot{\eta})^{2}+(\eta-\dot{\xi})^{2}\right)^{2} \\
&+\frac{3}{2 c^{2}}\left(\frac{q_{1}(1-\mu)}{r_{1}}+\frac{q_{2} \mu}{r_{2}}\right) \\
& \times\left((\xi+\dot{\eta})^{2}+(\eta-\dot{\xi})^{2}\right) \\
&-\frac{1}{2 c^{2}}\left(\frac{q_{1}(1-\mu)}{r_{1}}+\frac{q_{2} \mu}{r_{2}}\right)^{2}-\frac{q_{1} q_{2} \mu(1-\mu)}{2 c^{2}} \\
& \times\left[\frac{1}{r_{1}}+\left(\frac{1}{r_{1}}-\frac{1}{r_{2}}\right)(1-3 \mu-7 \xi-8 \dot{\eta})\right. \\
&\left.+\eta^{2}\left(\frac{q_{2} \mu}{r_{1}^{3}}+\frac{q_{1}(1-\mu)}{r_{2}^{3}}\right)\right], \\
& r_{1}=\sqrt{(\xi+\mu)^{2}+\eta^{2}}, \\
&(\xi+\mu-1)^{2}+\eta^{2}
\end{aligned}
$$

where $q_{i}=1-\left(F_{p} / F_{g}\right)_{i}, i=1,2$, is the mass reduction factor constant for a given particle. $F_{p}$, is the force due to radiation pressure, and $F_{g}$ is the force due to gravitational field, Sharma et al. [30], Simmons et al. [31], and Chernikov [32] with a modification to account for the radiation of the primaries $q_{i}$.

\section{Location of the Libration Points}

From the equations of motion (1), it is apparent that an equilibrium solution exists relative to the rotating frame when the partial derivatives of the pseudopotential function $\left(U_{\xi}, U_{\eta}, U_{\zeta}\right)$ are all zero; that is, $U=$ const. These points correspond to the positions in the rotating frame at which the gravitational forces and the centrifugal force associated with the rotation of the synodic frame all cancel, with the result that a particle located at one of these points appears stationary in the synodic frame. There are five equilibrium points in the circular RTBP, also known as Lagrange points or libration points. Three of them (the collinear points) lie along the $\xi$-axis: one interior point $L_{1}$ between the two primaries, and one point on the far side of each primary 


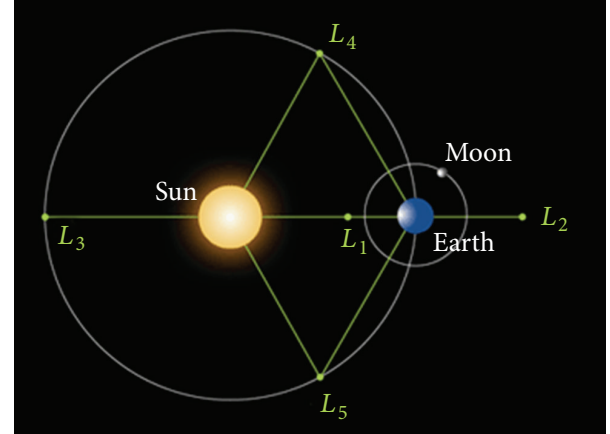

FIgURE 1: The five Lagrangian points with Sun-Earth system as an example.

with respect to the barycenter $L_{2}, L_{3}$. The other two libration points (the triangular points) are each located at the apex of an equilateral triangle formed with the primaries; see Figure 1. The triangular points are designated as $L_{4}$ and $L_{5}$, with $L_{4}$ moving ahead of the $\xi$-axis and $L_{5}$ trailing $\xi$-axis, along the orbit of the small primary as the synodic frame rotates uniformly relative to the inertial frame (as shown in Figure 1).

Remark 1. All the five libration points lie in the $\xi$ - $\eta$ plane, that is, in the plane of motion.

The libration points are obtained from equations of motion after setting $\ddot{\xi}=\ddot{\eta}=\dot{\xi}=\dot{\eta}=0$. These points represent particular solutions of equations of motion

$$
\begin{aligned}
& \frac{\partial U}{\partial \xi}=\frac{\partial U_{c+p h}}{\partial \xi}+\frac{\partial U_{r+\mathrm{ph}}}{\partial \xi}=0 \\
& \frac{\partial U}{\partial \eta}=\frac{\partial U_{c+\mathrm{ph}}}{\partial \eta}+\frac{\partial U_{r+\mathrm{ph}}}{\partial \eta}=0 .
\end{aligned}
$$

The explicit required partial derivatives are (remembering that $\left.U=U_{c+\mathrm{ph}}+U_{r+\mathrm{ph}}\right)$

$$
\begin{aligned}
& \frac{\partial U}{\partial \xi} \xi-\frac{q_{1}(1-\mu)(\xi+\mu)}{r_{1}^{3}}-\frac{q_{2} \mu(\xi+\mu-1)}{r_{2}^{3}}+\frac{1}{c^{2}} \\
& \times\left\{\left(\mu-\mu^{2}-3\right) \xi+\left(\frac{q_{1}(1-\mu)}{r_{1}}+\frac{q_{2} \mu}{r_{2}}\right)\right. \\
& \quad \times\left(\frac{q_{1}(1-\mu)(\xi+\mu)}{r_{1}^{3}}+\frac{q_{2} \mu(\xi+\mu-1)}{r_{2}^{3}}\right) \\
&+\frac{1}{2}\left(\eta^{2}+\xi^{2}\right) \xi-\frac{3}{2} \\
& \times\left(\frac{q_{1}(1-\mu)(\xi+\mu)}{r_{1}^{3}}+\frac{q_{2} \mu(\xi+\mu-1)}{r_{2}^{3}}\right) \\
& \quad \times\left(\eta^{2}+\xi^{2}\right)+3\left(\frac{q_{1}(1-\mu)}{r_{1}}+\frac{q_{2} \mu}{r_{2}}\right) \xi
\end{aligned}
$$

$$
\begin{aligned}
& -\frac{1}{2} q_{1} q_{2} \mu(1-\mu) \\
& \times\left[-\frac{1}{r_{1}^{3}}(\xi+\mu)\right. \\
& \quad+\left(\frac{(\xi+\mu)}{r_{1}^{3}}-\frac{(\xi+\mu-1)}{r_{2}^{3}}\right) \\
& \quad \times(-1+3 \mu+7 \xi)-7\left(\frac{1}{r_{1}}-\frac{1}{r_{2}}\right)-3 \eta^{2} \\
& \left.\left.\quad \times\left(\frac{q_{2} \mu(\xi+\mu)}{r_{1}^{5}}+\frac{q_{1}(1-\mu)(\xi+\mu-1)}{r_{2}^{5}}\right)\right]\right\}
\end{aligned}
$$

$=0$,

$$
\begin{aligned}
& \frac{\partial U}{\partial \eta} \\
& =\eta-\frac{q_{1}(1-\mu)}{r_{1}^{3}} \eta-\frac{q_{2} \mu}{r_{2}^{3}} \eta \\
& +\frac{1}{c^{2}}\left\{\left(\mu-\mu^{2}-3\right) \eta+\left(\frac{q_{1}(1-\mu)}{r_{1}}+\frac{q_{2} \mu}{r_{2}}\right)\right. \\
& \times\left(\frac{q_{1}(1-\mu)}{r_{1}^{3}}+\frac{q_{2} \mu}{r_{2}^{3}}\right) \eta+\frac{1}{2}\left(\xi^{2}+\eta^{2}\right) \eta \\
& -\frac{3}{2}\left(\xi^{2}+\eta^{2}\right)\left(\frac{q_{1}(1-\mu)}{r_{1}^{3}}+\frac{q_{2} \mu}{r_{2}^{3}}\right) \eta \\
& +3\left(\frac{q_{1}(1-\mu)}{r_{1}}+\frac{q_{2} \mu}{r_{2}}\right) \eta+\frac{q_{1} q_{2} \mu(1-\mu)}{2} \eta \\
& +\frac{1}{2} q_{1} q_{2} \mu(1-\mu) \eta \\
& \times\left[\left(-\frac{1}{r_{1}^{3}}+\frac{1}{r_{2}^{3}}\right)(-1+3 \mu+7 \xi)\right. \\
& +3\left(\frac{q_{1}(1-\mu)}{r_{2}^{5}}+\frac{q_{2} \mu}{r_{1}^{5}}\right) \eta^{2} \\
& \left.\left.+\frac{1}{r_{1}^{3}}-2\left(\frac{q_{2} \mu}{r_{1}^{3}}+\frac{q_{1}(1-\mu)}{r_{2}^{3}}\right)\right]\right\}=0 \text {. }
\end{aligned}
$$

\section{Location of Collinear Libration Points}

Any point of the collinear points must, by definition, have $\zeta=$ $\eta=0$, and the solution of the classical RTBP satisfies

$$
\begin{gathered}
\sigma_{1} r_{1}+\sigma_{2} r_{2}=1, \\
r_{1}=\sigma_{1}(\xi+\mu), \\
r_{2}=-\sigma_{2}(\mu+\xi-1),
\end{gathered}
$$


where (see Figures 2, 3, and 4) we have

$$
\begin{gathered}
L_{1}: \sigma_{1}=1, \sigma_{2}=1, \\
L_{2}: \sigma_{1}=1, \sigma_{2}=-1, \\
L_{3}: \sigma_{1}=-1, \sigma_{2}=1 .
\end{gathered}
$$

4.1. Location of $L_{1}$. Substituting from (8) with the corresponding values of $\sigma_{1}=1$ and $\sigma_{2}=1$ into (6) and noting that $\partial r_{1} / \partial \xi=-\partial r_{2} / \partial \xi=1$, we get

$$
\begin{aligned}
\frac{\partial U}{\partial \xi}= & -\left(\frac{q_{1}(1-\mu)}{r_{1}^{2}}-\frac{q_{2} \mu}{r_{2}^{2}}\right)+\frac{1}{c^{2}} \\
\times & \left\{\left(\mu-\mu^{2}-3\right) \xi+\left(\frac{q_{1}(1-\mu)}{r_{1}}+\frac{q_{2} \mu}{r_{2}}\right)\right. \\
& \times\left(\frac{q_{1}(1-\mu)}{r_{1}^{2}}-\frac{q_{2} \mu}{r_{2}^{2}}\right) \\
& -\frac{3}{2}\left(\frac{q_{1}(1-\mu)}{r_{1}^{2}}-\frac{q_{2} \mu}{r_{2}^{2}}\right) \xi^{2} \\
& +3\left(\frac{q_{1}(1-\mu)}{r_{1}}+\frac{q_{2} \mu}{r_{2}}\right) \xi+\frac{1}{2} \xi^{3} \\
& -\frac{1}{2} q_{1} q_{2} \mu(1-\mu) \\
& \times\left[-\frac{1}{r_{1}^{2}}+\left(\frac{1}{r_{1}^{2}}+\frac{1}{r_{2}^{2}}\right)(-1+3 \mu+7 \xi)\right. \\
& \left.\left.-7\left(\frac{1}{r_{1}}-\frac{1}{r_{2}}\right)\right]\right\}=0
\end{aligned}
$$

which can be re-written as a function of $r_{1}, r_{2}$ as

$$
\begin{aligned}
\frac{\partial U}{\partial \xi}=1-\mu & -r_{2}-\left(\frac{q_{1}(1-\mu)}{r_{1}^{2}}-\frac{q_{2} \mu}{r_{2}^{2}}\right) \\
+\frac{1}{c^{2}}\{ & \left(\frac{q_{1}(1-\mu)}{r_{1}}+\frac{q_{2} \mu}{r_{2}}\right)\left(\frac{q_{1}(1-\mu)}{r_{1}^{2}}-\frac{q_{2} \mu}{r_{2}^{2}}\right) \\
& +\left(\mu-\mu^{2}-3\right) \times\left(1-\mu-r_{2}\right) \\
& +\frac{1}{2}\left(1-\mu-r_{2}\right)^{3}-\frac{3}{2}\left(\frac{q_{1}(1-\mu)}{r_{1}^{2}}-\frac{q_{2} \mu}{r_{2}^{2}}\right) \\
& \times\left(1-\mu-r_{2}\right)^{2}+3\left(\frac{q_{1}(1-\mu)}{r_{1}}+\frac{q_{2} \mu}{r_{2}}\right)
\end{aligned}
$$

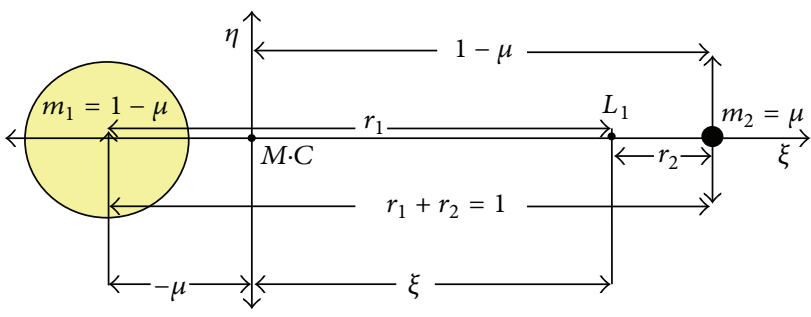

FIGURE 2: The Location of $L_{1}$.

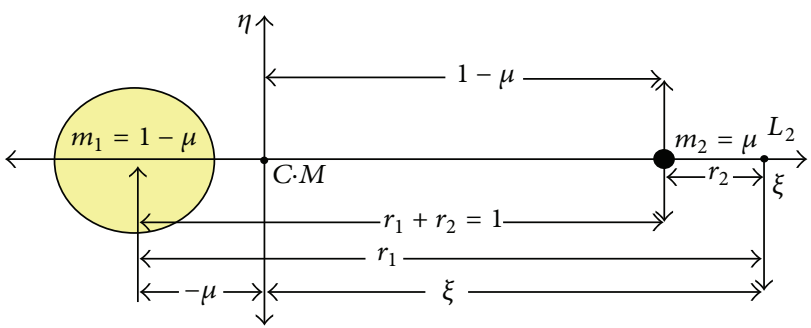

FIgure 3: The Location of $L_{2}$.

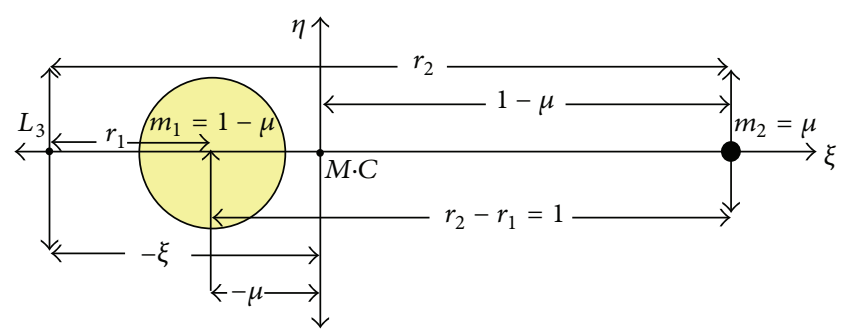

FIgURE 4: The Location of $L_{3}$.

$$
\begin{aligned}
& \times\left(1-\mu-r_{2}\right)-\frac{1}{2} q_{1} q_{2} \mu(1-\mu) \\
& \times\left[-\frac{1}{r_{1}^{2}}+\left(\frac{1}{r_{1}^{2}}+\frac{1}{r_{2}^{2}}\right)\right.
\end{aligned}
$$$$
\times\left(-1+3 \mu+7\left(1-\mu-r_{2}\right)\right)
$$

$$
\left.\left.-7\left(\frac{1}{r_{1}}-\frac{1}{r_{2}}\right)\right]\right\}=0 \text {. }
$$

Then it may be reasonable in our case to assume that positions of the equilibrium points $L_{1}$ are the same as given by classical RTBP but perturbed due to the inclusion of the relativistic correction by quantities $\varepsilon_{1} \equiv \mathcal{O}\left(1 / c^{2}\right)$. See Soffel [33] and Douskos and Perdios [7].

The solution of the classical part of (10) including the photogravitational correction can be obtained easily using the potential $U_{c+\mathrm{ph}}=q_{1}(1-\mu)\left(r_{1}^{2}+2 / r_{1}\right)+q_{2} \mu\left(r_{2}^{2}+2 / r_{2}\right)$ as follows:

$$
\begin{gathered}
\frac{\partial U_{c+\mathrm{ph}}}{\partial \xi}=\frac{\partial U_{c+\mathrm{ph}}}{\partial r_{1}} \frac{\partial r}{\partial \xi}+\frac{\partial U_{c+\mathrm{ph}}}{\partial r_{2}} \frac{\partial r_{2}}{\partial \xi}=0, \\
\Longrightarrow \frac{\partial U_{c+\mathrm{ph}}}{\partial \xi}=\frac{\partial U_{c+\mathrm{ph}}}{\partial r_{1}}-\frac{\partial U_{c+\mathrm{ph}}}{\partial r_{2}}=0
\end{gathered}
$$




$$
\begin{aligned}
& \Longrightarrow \frac{\partial U_{c+\mathrm{ph}}}{\partial r_{1}}=\frac{\partial U_{c+\mathrm{ph}}}{\partial r_{2}} \\
& \Longrightarrow \frac{q_{2} \mu}{q_{1}(1-\mu)}=\frac{\left(3 r_{2}-3 r_{2}^{2}+r_{2}^{3}\right) r_{2}^{2}}{\left(1-r_{2}\right)^{2}\left(1-r_{2}^{3}\right)}
\end{aligned}
$$

If $\left(q_{2} \mu\right) /\left(q_{1}(1-\mu)\right)$ is small, this equation has a root in the vicinity of $r_{2}=\alpha$, where $\alpha=\left(\left(q_{2} \mu\right) /\left(3 q_{1}(1-\mu)\right)\right)^{1 / 3}$; this $\alpha$ can be written as

$$
\alpha=r_{2}\left(1+\frac{1}{3} r_{2}+\frac{1}{3} r_{2}^{2}+\frac{53}{81} r_{2}^{3}+\frac{101}{243} r_{2}^{4}+\mathcal{O}\left(r_{2}^{5}\right)+\cdots\right) .
$$

Let successive approximations of $r_{2}$ be $r_{2 i}$ :

$$
\begin{gathered}
r_{20}=\alpha, \\
\alpha=r_{21}\left(1+\frac{1}{3} \alpha\right), \\
r_{21}=\alpha\left(1-\frac{1}{3} \alpha\right), \\
\alpha=r_{22}\left[1+\frac{1}{3} \alpha+\frac{2}{9} \alpha^{2}+\cdots\right],
\end{gathered}
$$

Then successive approximation yields

$$
r_{24}=\alpha\left(1-\frac{\alpha}{3}-\frac{\alpha^{2}}{9}+\frac{2}{27} \alpha^{3}+\frac{2}{81} \alpha^{4}+\mathcal{O}(\alpha)^{5}\right)
$$

Let us set the following notation:

$$
\begin{aligned}
& r_{1}=a_{1}+\varepsilon_{1}, \\
& r_{2}=b_{1}-\varepsilon_{1}, \\
& a_{1}=1-b_{1},
\end{aligned}
$$

where $a_{1}$ and $b_{1}$ are unperturbed relativistically positions of $r_{1}$ and $r_{2}$, respectively, and $b_{1}$ is given after some successive approximation by the relation

$$
\begin{aligned}
b_{1} & =r_{24} \\
& =\alpha\left(1-\frac{\alpha}{3}-\frac{\alpha^{2}}{9}+\frac{2}{27} \alpha^{3}+\frac{2}{81} \alpha^{4}+\mathcal{O}(\alpha)^{5}\right) .
\end{aligned}
$$

Substituting from (16) into (11) and retaining the terms up to the first order in the small quantities $\varepsilon_{1}$, we can get

$$
\begin{aligned}
& \frac{\partial U}{\partial \xi}=1-\mu-b_{1}+\varepsilon_{1} \\
& -\frac{q_{1}(1-\mu)}{\left(1-b_{1}\right)^{2}}\left(1-\frac{2 \varepsilon_{1}}{1-b_{1}}\right) \\
& +\frac{q_{2} \mu}{b_{1}^{2}}\left(1+\frac{2 \varepsilon_{1}}{b_{1}}\right)+\frac{1}{c^{2}} \\
& \times\left\{\left(\mu-\mu^{2}-3\right)\left(1-\mu-b_{1}\right)\right. \\
& +\left(\frac{q_{1}(1-\mu)}{\left(1-b_{1}\right)}+\frac{q_{2} \mu}{b_{1}}\right)\left(\frac{q_{1}(1-\mu)}{\left(1-b_{1}\right)^{2}}-\frac{q_{2} \mu}{b_{1}^{2}}\right) \\
& +\frac{1}{2}\left(1-\mu-b_{1}\right)^{3}-\frac{3}{2} \\
& \times\left(\frac{q_{1}(1-\mu)}{\left(1-b_{1}\right)^{2}}-\frac{q_{2} \mu}{b_{1}^{2}}\right) \times\left(1-\mu-b_{1}\right)^{2} \\
& +3\left(\frac{q_{1}(1-\mu)}{\left(1-b_{1}\right)}+\frac{q_{2} \mu}{b_{1}}\right) \\
& \times\left(1-\mu-b_{1}\right)-\frac{1}{2} q_{1} q_{2} \mu(1-\mu) \\
& \times\left[-\frac{1}{\left(1-b_{1}\right)^{2}}-7\left(\frac{1}{\left(1-b_{1}\right)}-\frac{1}{b_{1}}\right)\right. \\
& +\left(\frac{1}{\left(1-b_{1}\right)^{2}}+\frac{1}{b_{1}^{2}}\right) \\
& \left.\left.\times\left(6-7 b_{1}-4 \mu\right)\right]\right\}=0 \text {. }
\end{aligned}
$$

Equation (18) can be solved for $\varepsilon_{1}$; then substituting back into (16) and (8) yields, after some lengthy algebraic manipulations, the location of $L_{1}$ as

$$
\begin{aligned}
\xi_{0, L_{1}}= & 1-\mu \\
& -\sum_{k=0}^{5}\left[\mathscr{M}_{k}^{L_{1}}+\left(\frac{1}{c^{2}}\right) \mathcal{N}_{k}^{L_{1}}\right]\left(\frac{\mu}{3}\right)^{k / 3}+\mathcal{O}\left(\frac{\mu}{3}\right)^{2},
\end{aligned}
$$

where the nonvanishing coefficients $\mathscr{M}_{k}^{L_{1}}$ and $\mathcal{N}_{k}^{L_{1}}$, are collected in Appendix A.

4.2. Location of $L_{2}$. The collinear point $L_{2}$ is located outside the small massive primary of mass $\mu$. We now drive an approximate location for this point under the considered perturbations. Substituting from (8) with the corresponding 
values of $\sigma_{1}=1$ and $\sigma_{2}=-1$ into (6) and noting that $\partial r_{1} / \partial \xi=\partial r_{2} / \partial \xi=1$, we get

$$
\begin{gathered}
r_{1}-r_{2}=1, \\
r_{1}=\xi+\mu, \\
r_{2}=\xi+\mu-1, \\
\frac{\partial r_{1}}{\partial \xi}=\frac{\partial r_{2}}{\partial \xi}=1 .
\end{gathered}
$$

Substituting from (20) into (6), we get

$$
\begin{aligned}
\frac{\partial U}{\partial \xi}=\xi & \left(\frac{q_{1}(1-\mu)}{r_{1}^{2}}-\frac{q_{2} \mu}{r_{2}^{2}}\right)+\frac{1}{c^{2}} \\
\times & \left\{\left(\mu-\mu^{2}-3\right) \xi+\left(\frac{q_{1}(1-\mu)}{r_{1}}+\frac{q_{2} \mu}{r_{2}}\right)\right. \\
& \times\left(\frac{q_{1}(1-\mu)}{r_{1}^{2}}-\frac{q_{2} \mu}{r_{2}^{2}}\right) \\
& -\frac{3}{2}\left(\frac{q_{1}(1-\mu)}{r_{1}^{2}}-\frac{q_{2} \mu}{r_{2}^{2}}\right) \xi^{2} \\
& +3\left(\frac{q_{1}(1-\mu)}{r_{1}}+\frac{q_{2} \mu}{r_{2}}\right) \xi+\frac{1}{2} \xi^{3} \\
& -\frac{1}{2} q_{1} q_{2} \mu(1-\mu) \\
& \times\left[-\frac{1}{r_{1}^{2}}+\left(\frac{1}{r_{1}^{2}}-\frac{1}{r_{2}^{2}}\right)\right. \\
& \left.\left.\times(-1+3 \mu+7 \xi)-7\left(\frac{1}{r_{1}}-\frac{1}{r_{2}}\right)\right]\right\}=0
\end{aligned}
$$

which can be rewritten as a function of $r_{1}$ and $r_{2}$ as

$$
\begin{aligned}
\frac{\partial U}{\partial \xi}=1-\mu+ & r_{2}-\left(\frac{q_{1}(1-\mu)}{r_{1}^{2}}-\frac{q_{2} \mu}{r_{2}^{2}}\right) \\
+\frac{1}{c^{2}}\{( & \left(\mu-\mu^{2}-3\right) \xi+\left(\frac{q_{1}(1-\mu)}{r_{1}}+\frac{q_{2} \mu}{r_{2}}\right) \\
& \times\left(\frac{q_{1}(1-\mu)}{r_{1}^{2}}-\frac{q_{2} \mu}{r_{2}^{2}}\right) \\
& -\frac{3}{2}\left(\frac{q_{1}(1-\mu)}{r_{1}^{2}}-\frac{q_{2} \mu}{r_{2}^{2}}\right) \xi^{2} \\
+ & 3\left(\frac{q_{1}(1-\mu)}{r_{1}}+\frac{q_{2} \mu}{r_{2}}\right) \xi+\frac{1}{2} \xi^{3}
\end{aligned}
$$

$$
\begin{aligned}
& -\frac{1}{2} q_{1} q_{2} \mu(1-\mu) \\
& \times\left[-\frac{1}{r_{1}^{2}}+\left(\frac{1}{r_{1}^{2}}-\frac{1}{r_{2}^{2}}\right)\right. \\
& \left.\left.\quad \times(-1+3 \mu+7 \xi)-7\left(\frac{1}{r_{1}}-\frac{1}{r_{2}}\right)\right]\right\}=0 .
\end{aligned}
$$

Then, it may be reasonable in our case to assume that the position of the equilibrium point $L_{2}$ is the same as given by classical RTBP but perturbed due to the inclusion of the relativistic correction by quantities $\varepsilon_{2} \equiv \mathcal{O}\left(1 / c^{2}\right)$ :

$$
\begin{aligned}
& r_{1}=a_{2}+\varepsilon_{2}, \\
& r_{2}=b_{2}+\varepsilon_{2}, \\
& a_{2}=1+b_{2}
\end{aligned}
$$

with $a_{2}$ and $b_{2}$ being the unperturbed positions of $r_{1}$ and $r_{2}$, respectively, where $b_{2}$ is given after some successive approximation as

$$
\begin{gathered}
b_{2}=\alpha\left(1+\frac{\alpha}{3}-\frac{\alpha^{2}}{9}-\frac{2}{27} \alpha^{3}+\frac{2}{81} \alpha^{4}+\mathcal{O}(\alpha)^{5}\right), \\
\alpha=\left(\frac{q_{2} \mu}{3 q_{1}(1-\mu)}\right)^{1 / 3} .
\end{gathered}
$$

Substituting from (23) into (22) and retaining the terms up to the first order in the small quantities $\varepsilon_{2}$, we can get

$$
\begin{aligned}
\frac{\partial U}{\partial \xi}=1-\mu+ & b_{2}+\varepsilon_{2}-\frac{q_{1}(1-\mu)}{\left(1+b_{2}\right)^{2}} \\
& \times\left(1-\frac{2 \varepsilon_{2}}{1+b_{2}}\right)-\frac{q_{2} \mu}{b_{2}^{2}}\left(1-\frac{2 \varepsilon_{2}}{b_{2}}\right) \\
+\frac{1}{c^{2}}\{ & \left(\mu-\mu^{2}-3\right)\left(1-\mu+b_{2}\right) \\
& +\left(\frac{q_{1}(1-\mu)}{\left(1+b_{2}\right)}+\frac{q_{2} \mu}{b_{2}}\right) \\
& \times\left(\frac{q_{1}(1-\mu)}{\left(1+b_{2}\right)^{2}}+\frac{q_{2} \mu}{b_{2}^{2}}\right) \\
& +\frac{1}{2}\left(1-\mu+b_{2}\right)^{3} \\
& -\frac{3}{2}\left(\frac{q_{1}(1-\mu)}{\left(1+b_{2}\right)^{2}}+\frac{q_{2} \mu}{b_{2}^{2}}\right) \\
& \times\left(\frac{q_{1}(1-\mu)}{\left(1+b_{2}\right)}+\frac{q_{2} \mu}{b_{2}}\right) \\
&
\end{aligned}
$$




$$
\begin{aligned}
& \times\left(1-\mu+b_{2}\right)-\frac{1}{2} q_{1} q_{2} \mu(1-\mu) \\
& \times\left[-\frac{1}{\left(1+b_{2}\right)^{2}}-7\left(\frac{1}{\left(1+b_{2}\right)}-\frac{1}{b_{2}}\right)\right. \\
& \left.\left.\quad+\left(\frac{1}{\left(1+b_{2}\right)^{2}}-\frac{1}{b_{2}^{2}}\right)\left(6+7 b_{2}-4 \mu\right)\right]\right\}
\end{aligned}
$$$$
=0 \text {. }
$$

Equation (25) can be solved for $\varepsilon_{2}$; then substituting back into (23) and (20) yields, after some lengthy algebraic manipulations, the location of $L_{2}$ as

$$
\begin{aligned}
\xi_{0, L_{2}}= & 1-\mu \\
& +\sum_{k=0}^{5}\left[\mathscr{M}_{k}^{L_{2}}+\left(\frac{1}{c^{2}}\right) \mathcal{N}_{k}^{L_{2}}\right]\left(\frac{\mu}{3}\right)^{k / 3} \\
& +\mathcal{O}\left(\frac{\mu}{3}\right)^{2},
\end{aligned}
$$

where the non-vanishing coefficients $\mathscr{M}_{k}^{L_{2}}$ and $\mathcal{N}_{k}^{L_{2}}$, are collected in Appendix B.

4.3. Location of $L_{3}$. The $L_{3}$ point lies on the negative $\xi$ axis. We now derive an approximate location for this point. Substituting from (8) with the corresponding values of $\sigma_{1}=$ -1 and $\sigma_{2}=1$ into $(6)$ and noting that $\partial r_{1} / \partial \xi=\partial r_{2} / \partial \xi=-1$, we get

$$
\begin{gathered}
r_{2}-r_{1}=1, \\
r_{1}=-(\xi+\mu), \\
r_{2}=1-\mu-\xi, \\
\frac{\partial r_{1}}{\partial \xi}=\frac{\partial r_{2}}{\partial \xi}=-1 .
\end{gathered}
$$

Hence substituting from (27) into (6), we get

$$
\begin{aligned}
\frac{\partial U}{\partial \xi}=\xi+\left(\frac{q_{1}(1-\mu)}{r_{1}^{2}}+\frac{q_{2} \mu}{r_{2}^{2}}\right) \\
+\frac{1}{c^{2}}\left\{\left(\mu-\mu^{2}-3\right) \xi-\left(\frac{q_{1}(1-\mu)}{r_{1}}+\frac{q_{2} \mu}{r_{2}}\right)\right. \\
+\left(\frac{q_{1}(1-\mu)}{r_{1}^{2}}+\frac{q_{2} \mu}{r_{2}^{2}}\right) \\
+\frac{1}{2} \xi^{3}+\frac{3}{2}\left(\frac{q_{1}(1-\mu)}{r_{1}^{2}}+\frac{q_{2} \mu}{r_{2}^{2}}\right) \xi^{2} \\
+3\left(\frac{q_{1}(1-\mu)}{r_{1}}+\frac{q_{2} \mu}{r_{2}}\right) \xi
\end{aligned}
$$

$$
\begin{gathered}
-\frac{1}{2} q_{1} q_{2} \mu(1-\mu) \\
\times\left[\frac{1}{r_{1}^{2}}-\left(\frac{1}{r_{1}^{2}}-\frac{1}{r_{2}^{2}}\right)(-1+3 \mu+7 \xi)\right. \\
\left.\left.-7\left(\frac{1}{r_{1}}-\frac{1}{r_{2}}\right)\right]\right\}=0
\end{gathered}
$$

which can be rewritten as a function of $r_{1}$ and $r_{2}$ as

$$
\begin{aligned}
\frac{\partial U}{\partial \xi} & =r_{2}+\left(\frac{q_{1}(1-\mu)}{r_{1}^{2}}+\frac{q_{2} \mu}{r_{2}^{2}}\right) \\
+\frac{1}{c^{2}}\{( & \left.\mu-\mu^{2}-3\right)\left(1-\mu-r_{2}\right) \\
& -\left(\frac{q_{1}(1-\mu)}{r_{1}}+\frac{q_{2} \mu}{r_{2}}\right) \\
& \times\left(\frac{q_{1}(1-\mu)}{r_{1}^{2}}+\frac{q_{2} \mu}{r_{2}^{2}}\right) \\
+ & \frac{1}{2}\left(1-\mu-r_{2}\right)^{3} \\
+ & \frac{3}{2}\left(\frac{q_{1}(1-\mu)}{r_{1}^{2}}+\frac{q_{2} \mu}{r_{2}^{2}}\right) \\
& \times\left(1-\mu-r_{2}\right)^{2} \\
& +3 \frac{1}{2} q_{1} q_{2} \mu(1-\mu) \\
& \left.+\frac{q_{1}(1-\mu)}{r_{1}}+\frac{q_{2} \mu}{r_{2}}\right)\left(1-\mu-r_{2}\right)
\end{aligned}
$$

Then it may be reasonable in our case to assume that the position of the equilibrium point $L_{3}$ is the same as given by classical restricted three-body problem but perturbed due to the inclusion of the relativistic correction by quantities $\varepsilon_{3} \equiv \mathcal{O}\left(1 / c^{2}\right)$ :

$$
\begin{aligned}
& r_{1}=a_{3}+\varepsilon_{3}, \\
& r_{2}=b_{3}+\varepsilon_{3}, \\
& a_{3}=b_{3}-1,
\end{aligned}
$$


where $a_{3}$ and $b_{3}$ are the unperturbed values of $r_{1}$ and $r_{2}$, respectively, and $b_{3}$ is given after some successive approximation by the relation

$$
\begin{aligned}
b_{3}= & 2-\frac{7}{4}\left(\frac{q_{2}}{q_{1}}\right)\left(\frac{\mu}{3}\right)+\frac{21\left(-q_{1}+q_{2}\right) \mu^{2}}{4 q_{2}}\left(\frac{q_{2}}{q_{1}}\right)^{2}\left(\frac{\mu}{3}\right)^{2} \\
& +\frac{147\left(-q_{1}+q_{2}\right)}{16 q_{2}}\left(\frac{q_{2}}{q_{1}}\right)^{3}\left(\frac{\mu}{3}\right)^{3} \\
& +\frac{1029\left(-q_{1}+q_{2}\right)}{64 q_{2}}\left(\frac{q_{2}}{q_{1}}\right)^{4}\left(\frac{\mu}{3}\right)^{4} \\
& -\frac{7203\left(q_{1}-q_{2}\right)}{256 q_{2}}\left(\frac{q_{2}}{q_{1}}\right)^{5}\left(\frac{\mu}{3}\right)^{5}+\mathcal{O}\left(\frac{\mu}{3}\right)^{6} .
\end{aligned}
$$

Substituting from (30) into (29) and retaining the terms up to the first order in the small quantities $\varepsilon_{3}$, we can get

$$
\begin{aligned}
& \frac{\partial U}{\partial \xi} \\
& =1-\mu-b_{3}-\varepsilon_{3}+\frac{q_{1}(1-\mu)}{\left(1-b_{3}\right)^{2}} \\
& \times\left(1+\frac{2 \varepsilon_{3}}{1-b_{3}}\right)+\frac{q_{2} \mu}{b_{3}^{2}}\left(1-\frac{2 \varepsilon_{3}}{b_{3}}\right)+\frac{1}{c^{2}} \\
& \times\left\{\left(\mu-\mu^{2}-3\right)\left(1-\mu-b_{3}\right)\right. \\
& -\left(\frac{q_{1}(1-\mu)}{\left(1-b_{3}\right)}-\frac{q_{2} \mu}{b_{3}}\right)\left(\frac{q_{1}(1-\mu)}{\left(1-b_{3}\right)^{2}}+\frac{q_{2} \mu}{b_{3}^{2}}\right) \\
& +\frac{1}{2}\left(1-\mu-b_{3}\right)^{3}+\frac{3}{2}\left(\frac{q_{1}(1-\mu)}{\left(1-b_{3}\right)^{2}}+\frac{q_{2} \mu}{b_{3}^{2}}\right) \\
& \times\left(1-\mu-b_{3}\right)^{2}-3\left(\frac{q_{1}(1-\mu)}{\left(1-b_{3}\right)}-\frac{q_{2} \mu}{b_{3}}\right) \\
& \times\left(1-\mu-b_{3}\right)-\frac{1}{2} q_{1} q_{2} \mu(1-\mu) \\
& \times\left[\frac{1}{\left(1-b_{3}\right)^{2}}-\left(\frac{1}{\left(1-b_{3}\right)^{2}}-\frac{1}{b_{3}^{2}}\right)\right. \\
& \left.\left.\times\left(6-7 b_{3}-4 \mu\right)-7\left(\frac{1}{\left(1-b_{3}\right)}-\frac{1}{b_{3}}\right)\right]\right\}=0 \text {. }
\end{aligned}
$$

Equation (32) can be solved for $\varepsilon_{3}$; then substituting back into (30) and (27) yields, after some lengthy algebraic manipulations, the location of $L_{3}$ as

$$
\xi_{0, L_{3}}=1-\mu-\sum_{k=0}^{5}\left[\mathscr{M}_{k}^{L_{3}}+\left(\frac{1}{c^{2}}\right) \mathcal{N}_{k}^{L_{3}}\right]\left(\frac{\mu}{3}\right)^{k}+\mathcal{O}\left(\frac{\mu}{3}\right)^{6}
$$

where the non-vanishing coefficients $\mathscr{M}_{k}^{L_{3}}$ and $\mathcal{N}_{k}^{L_{3}}$ are collected in Appendix C.

Remark 2. It is worth noting that there is no contribution in other two coordinates; that is, $\eta_{0, L_{i}}=\zeta_{0, L_{i}}=0, i=1,2,3$. This can be interpreted due to the fact that the concerned relativistic field is conservative.

\section{Numerical Representations and Analyses}

In this section we design a Mathematica program to draw the locations of collinear points $L_{i}, i=1,2,3$, versus the whole range of the mass ratio $\mu$ taking into account the photogravitational effects and/or the relativistic corrections. In the case of one body radiates we considering the following values of the photogravitational parameter: $q_{i}=1,0.8,0.6,0.4,0.2, i=$ 1,2 , and they are represented on figures as black (no radiation exist), green, blue, orange, and red, respectively. But in the case two primaries radiating we adopted the following values, $q_{i}=1,0.6,0.2, i=1,2$, and they are black (no radiation exist), blue, and red, respectively, this is actually to avoid the high density of the curves represented on these figures.

5.1. Analysis of $L_{1}$ Location. The black curve represents the case when the radiation pressure is totally neglected. In Figures 5(a) and 5(b), it seems that when $\mu=0.5$ the location of $L_{1}$ lies exactly in the barycenter, and when $\mu \rightarrow 0$ the location of $L_{1}$ is shifted towards the center of the less massive primary and then the problem reduced to the classical twobody problem. The same case also exists in Figure 5(c) when there is no radiation from the two primaries, but when one or two primaries radiate, the situation is changed.

In Figure 5(a), we plot the location of $L_{1}$ under the effect of changing $q_{1}$ (the radiation coefficient of the massive primary $1-\mu$ ) with different mass ratios. The green, blue, orange, and red curves represent the effect of successive increase in radiation of the massive primary, respectively. As is cleared from these curves illustrate, the greater the effect of the radiation (mass loss) the greater the negative displacement of the location of $L_{1}$ away from less massive primary, that is, towards the massive primary. This effect is of nonlinear nature. When the mass loss is significantly large especially in the case of binary systems of comparable masses (near $\mu=0.4$ and greater), the location of $L_{1}$ crosses the barycenter towards the massive primary, that is, along the negative $\xi$-axis.

In Figure 5(b) we plot the location of $L_{1}$ under the effect of changing $q_{2}$ (the radiation coefficient of the less massive primary $\mu$ ) with different mass ratios; the greater the effect of the radiation (mass loss) the greater the positive displacement of the location of $L_{1}$ towards less massive primary. When the mass loss is significantly large especially in the case of binary systems of comparable masses (near $\mu=0.4$ and greater), the location of $L_{1}$ never crosses the barycenter towards the less massive primary. This may be interpreted as follows: as the emitter in this case is the less massive body, within the domain of classical stability $\mu \in(0,0.038)$ the location of $L_{1}$ is not affected so much in contrast to the greater mass ratios. 


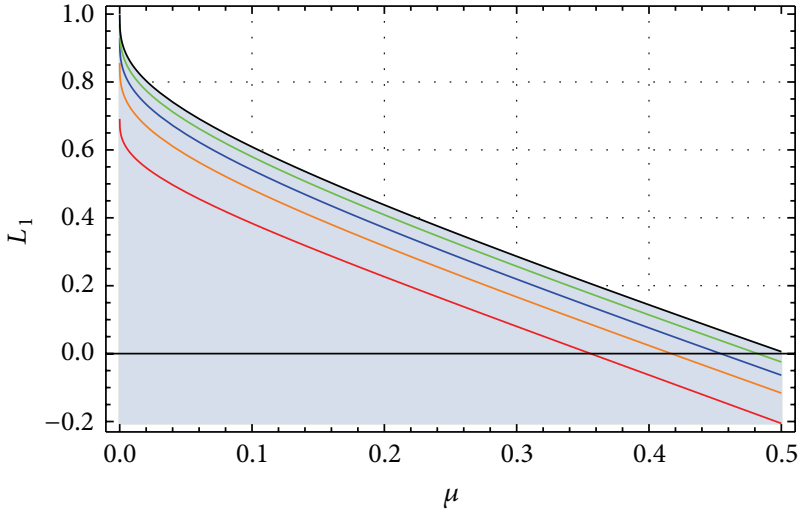

(a)

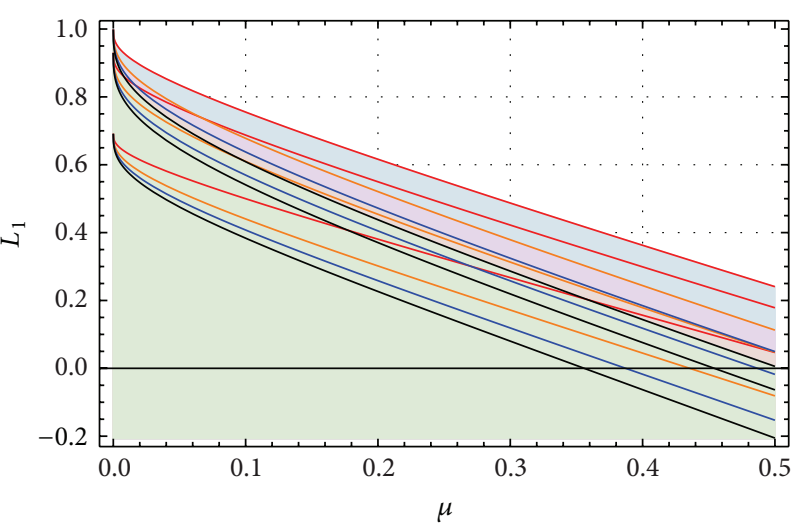

(c)

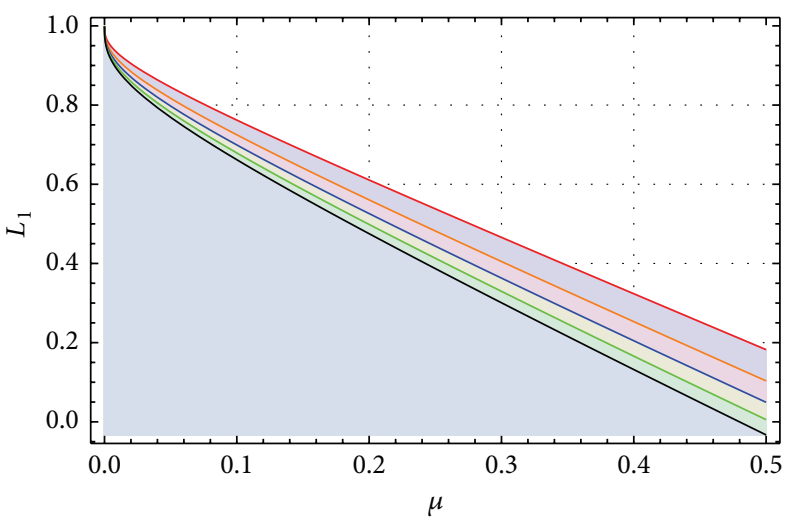

(e)

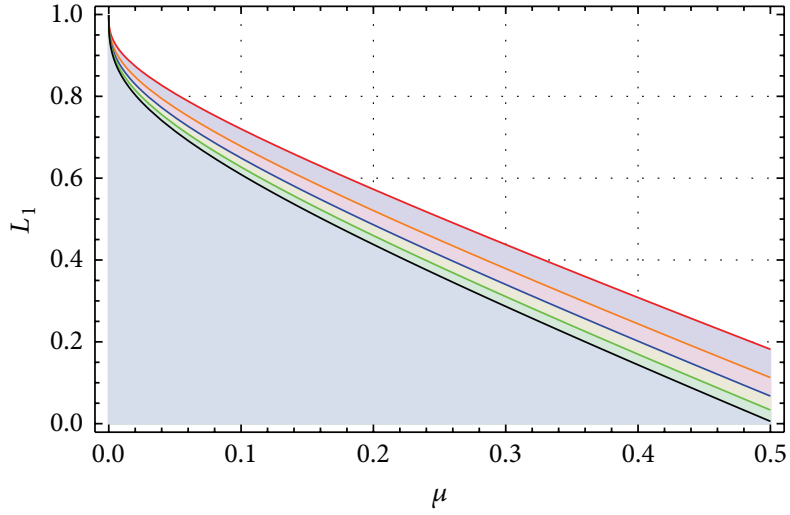

(b)

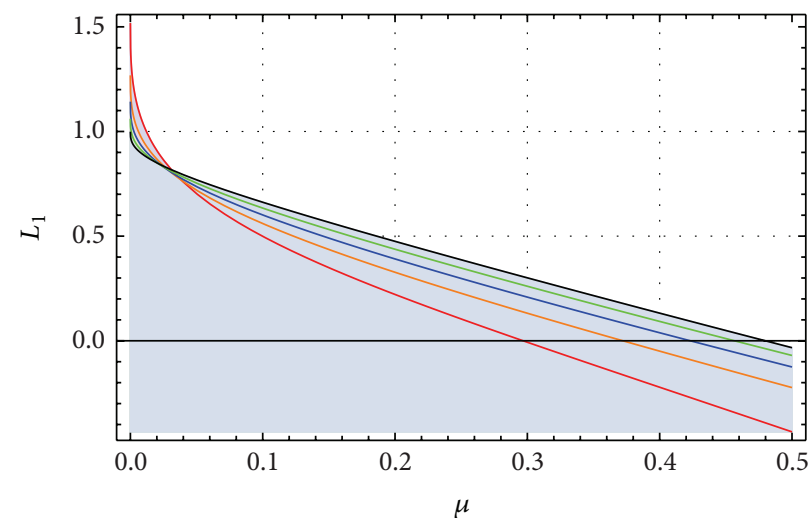

(d)

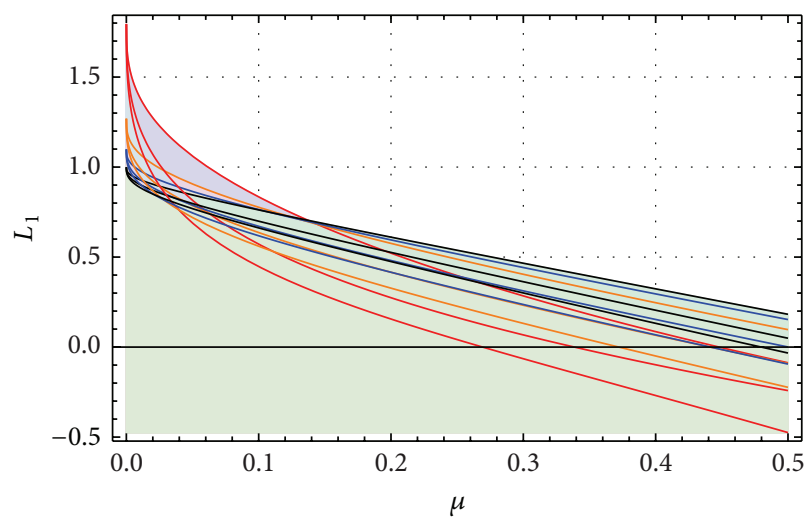

(f)

FIGURE 5: (a) The location of $L_{1}$ against mass ratio $\mu$ under the effect of changing $q_{1}$ and constant $q_{2}$ without relativistic corrections. (b) The location of $L_{1}$ against mass ratio $\mu$ under the effect of changing $q_{2}$ and constant $q_{1}$ without relativistic corrections. (c) The location of $L_{1}$ against mass ratio $\mu$ under the effect of changing $q_{1}$ and $q_{2}$ without relativistic corrections. (d) The location of $L_{1}$ against mass ratio $\mu$ under the effect of changing $q_{1}$ and constant $q_{2}$ with relativistic corrections. (e) The location of $L_{1}$ against mass ratio $\mu$ under the effect of changing $q_{2}$ and constant $q_{1}$ with relativistic corrections. (f) The location of point $L_{1}$ against mass ratio $\mu$ under the effect of changing $q_{1}$ and $q_{2}$ with relativistic corrections.

In Figure 5(c), we plot the location of $L_{1}$ under the effect of changing $q_{1}$ and $q_{2}$ (the radiation coefficient of the two primaries). The situation is a combination of the two effects depicted in Figures 5(a) and 5(b). Since the point $L_{1}$ is closer to less massive primary, the effect of the radiation of it is dominant. With the observation that when the mass loss is significantly large especially in the case of binary systems of comparable masses (near $\mu=0.4$ and greater), the location of $L_{1}$ crosses the barycenter towards the massive primary.

Figures 5(d)-5(f) represent the location of Lagrangian point $L_{1}$ under the effect of changing $q_{1}$ and/or $q_{2}$ with relativistic corrections. In Figure 5(d) we plot the location of $L_{1}$ under the effect of changing $q_{1}$ (the radiation coefficient of the less massive primary $\mu$ ) with relativistic corrections 


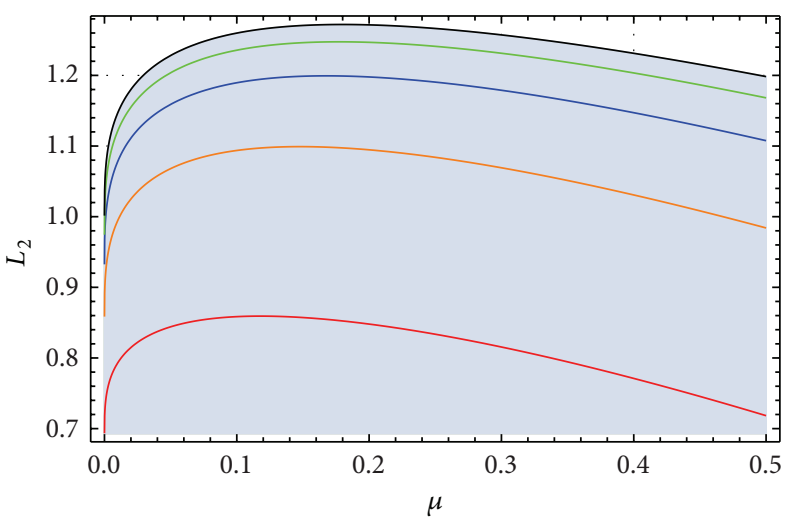

(a)

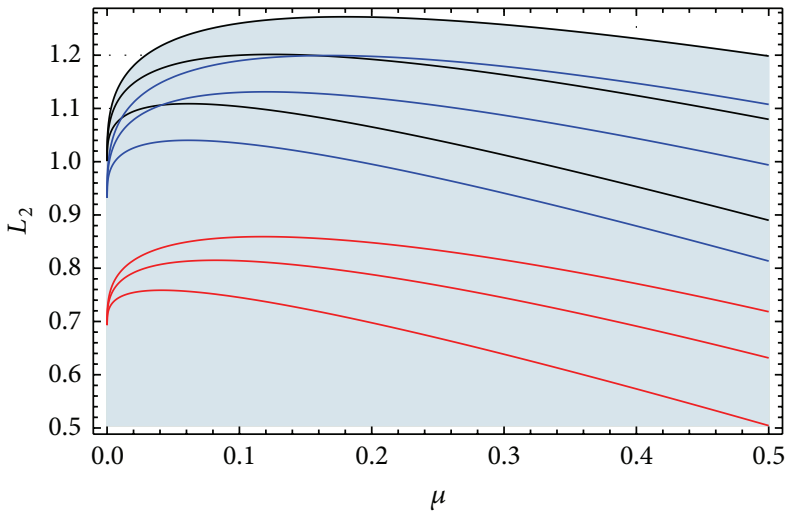

(c)

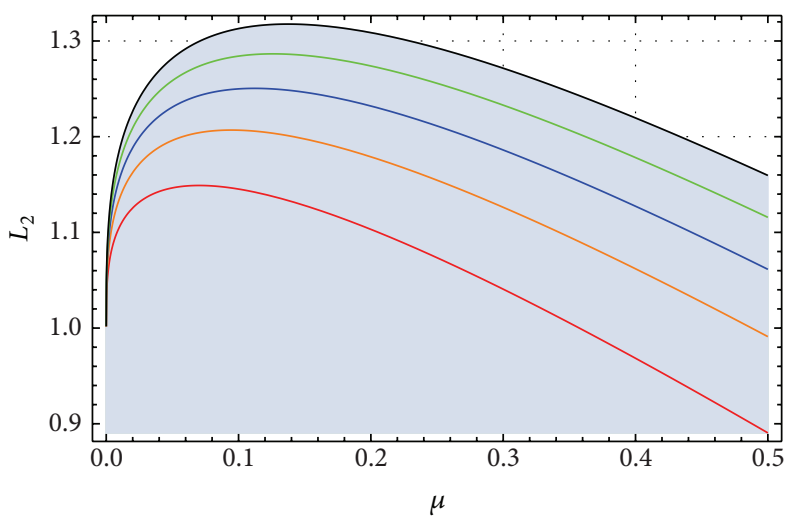

(e)

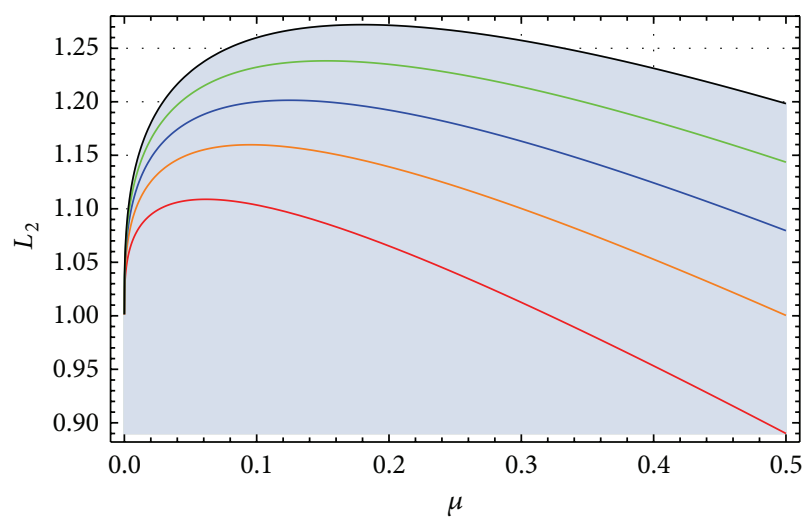

(b)

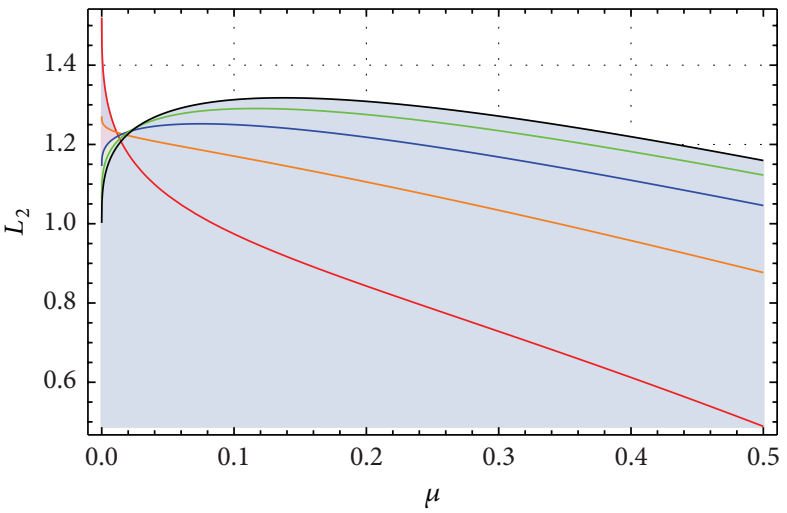

(d)

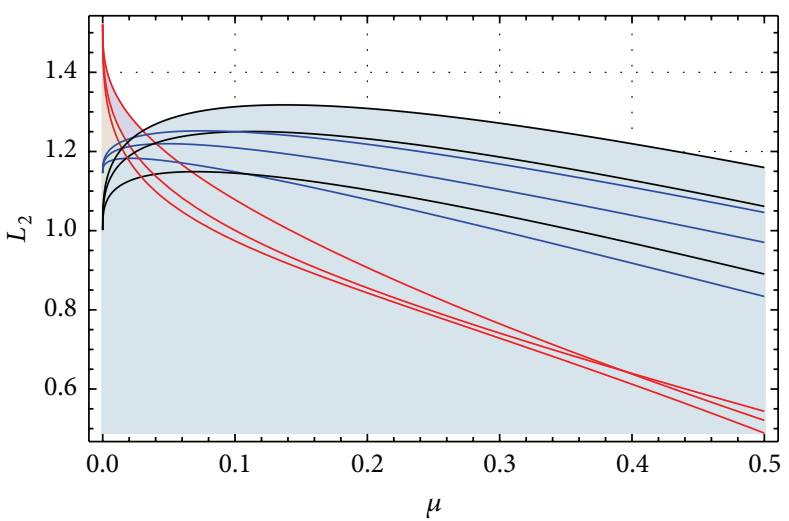

(f)

FIgURE 6: (a) The location of $L_{2}$ against mass ratio $\mu$ under the effect of changing $q_{1}$ and constant $q_{2}$ without relativistic corrections. (b) The location of $L_{2}$ against mass ratio $\mu$ under the effect of changing $q_{2}$ and constant $q_{1}$ without relativistic corrections. (c) The location of $L_{2}$ against mass ratio $\mu$ under the effect of changing $q_{1}$ and $q_{2}$ without relativistic corrections. (d) The location of $L_{2}$ against mass ratio $\mu$ under the effect of changing $q_{1}$ and constant $q_{2}$ with relativistic corrections. (e) The location of $L_{2}$ against mass ratio $\mu$ under the effect of changing $q_{2}$ and constant $q_{1}$ with relativistic corrections. (f) The location of $L_{2}$ against mass ratio $\mu$ under the effect of changing $q_{1}$ and $q_{2}$ with relativistic corrections.

versus different mass ratios. The greater the effect of the radiation (mass loss), the greater the positive displacement of the location of $L_{1}$ towards less massive primary in the domain $\mu \in(0,0.038)$. The most observable thing is that $L_{1}$ crosses to the right of the less massive primary especially in the very small mass ratios. Beyond this domain, that is, when $\mu \in(0.038,0.5)$, the situation converses, but the nonlinearity becomes more visible. In Figure 5(e), the dynamics is similar to that in Figure 5(b) but with clear different sizes of perturbations. In Figure 5(f), we have combined effects of those depicted in Figures 5(d) and 5(e).

5.2. Analysis of $L_{2}$ Location. The black curve represents the case when the radiation pressure is totally neglected. In Figures 6(a) and 6(b), it seems that when $\mu=0.5$ the location of $L_{2}$ lies exactly at 1.2 , and when $\mu \rightarrow 0$ the location of 


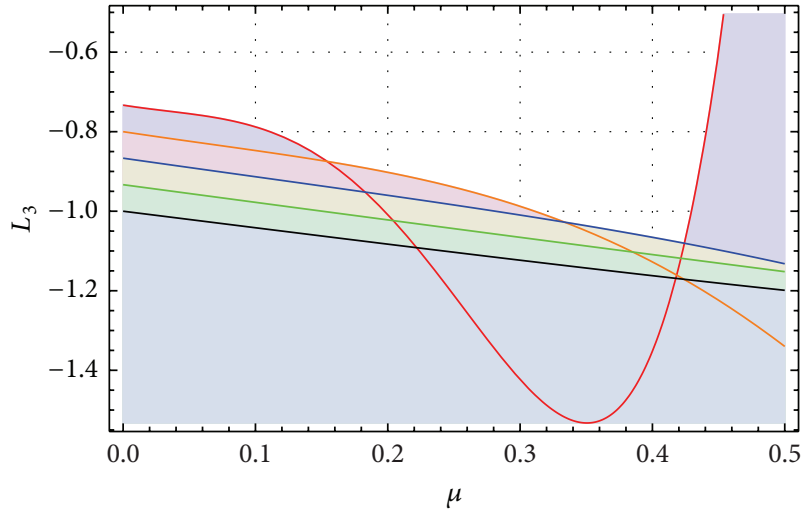

(a)

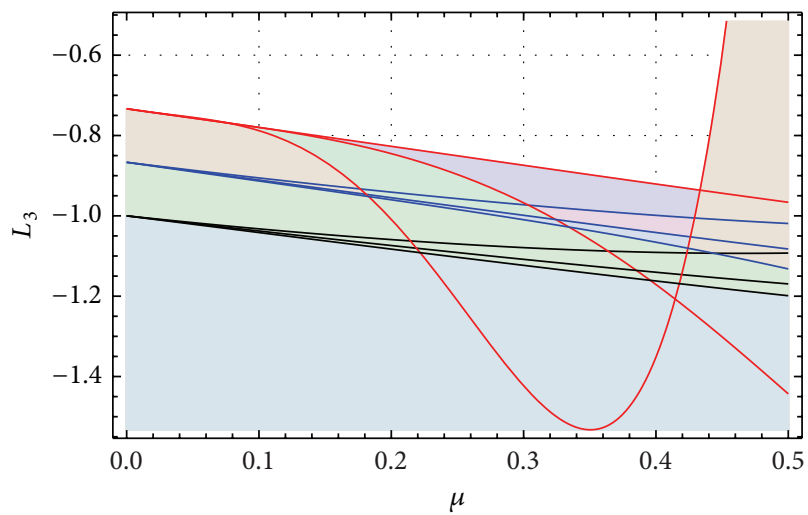

(c)

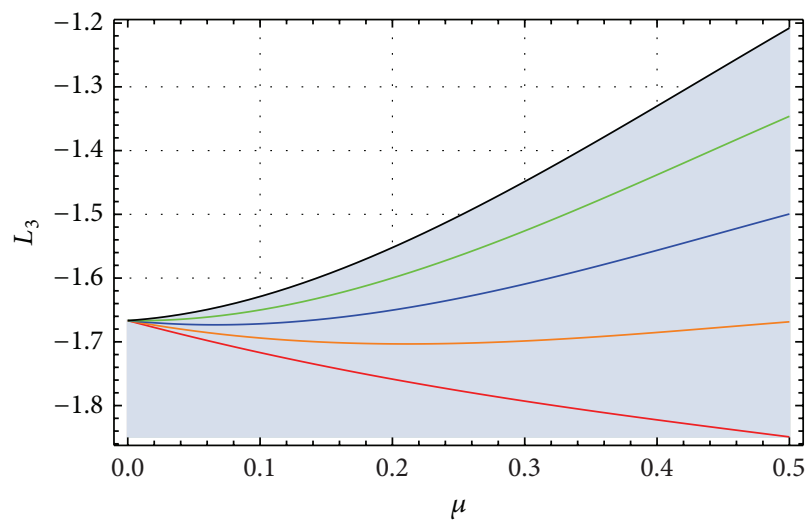

(e)

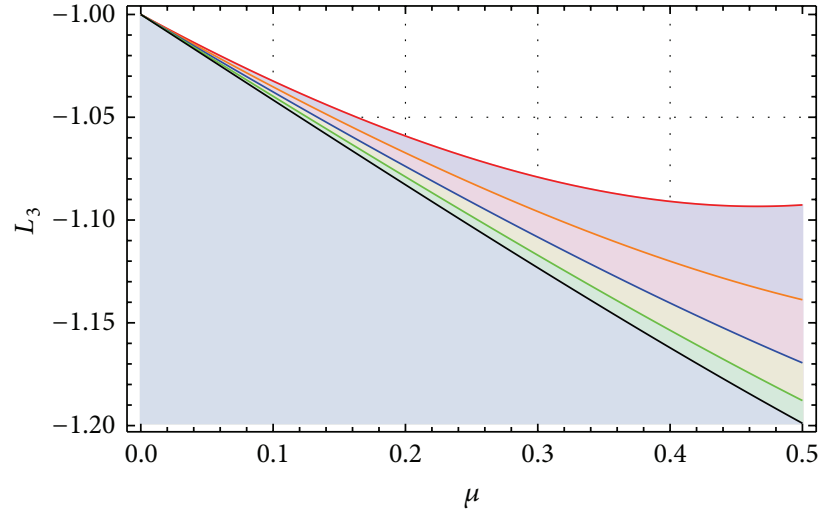

(b)

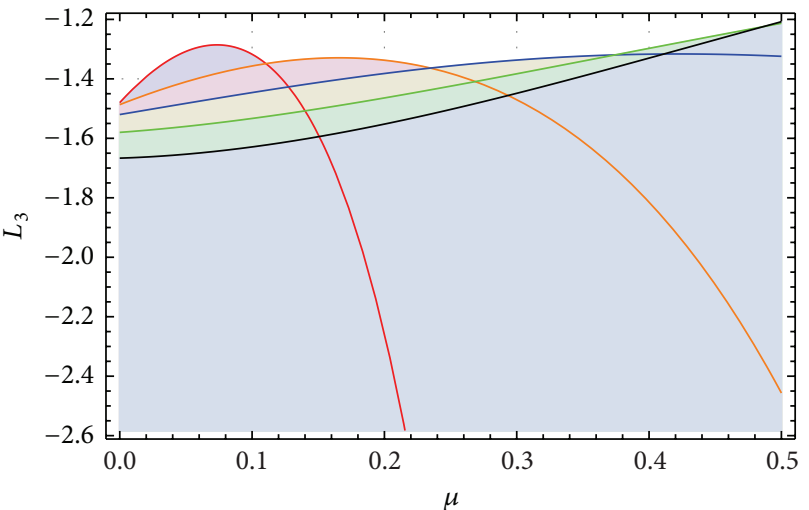

(d)

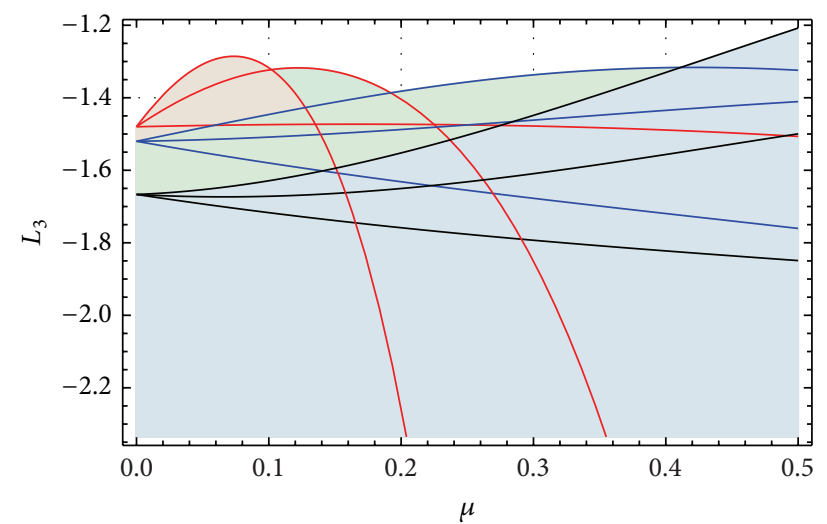

(f)

Figure 7: (a) The location of $L_{3}$ against mass ratio $\mu$ under the effect of changing $q_{1}$ and constant $q_{2}$ without relativistic corrections. (b) The location of $L_{3}$ against mass ratio $\mu$ under the effect of changing $q_{2}$ and constant $q_{1}$ without relativistic corrections. (c) The location of $L_{3}$ against mass ratio $\mu$ under the effect of changing $q_{1}$ and $q_{2}$ without relativistic corrections. (d) The location of $L_{1}$ against mass ratio $\mu$ under the effect of changing $q_{1}$ and constant $q_{2}$ with relativistic corrections. (e) The location of $L_{3}$ against mass ratio $\mu$ under the effect of changing $q_{2}$ and constant $q_{1}$ with relativistic corrections. (f) The location of $L_{3}$ against mass ratio $\mu$ under the effect of changing $q_{1}$ and $q_{2}$ with relativistic corrections.

$L_{2}$ is shifted to the left, that is, towards the center of the less massive primary and lies exactly at a normalized distance equal to 1 on the right of the barycenter. Then the problem reduced to the classical two body problem. The same case also exists in Figure 6(c) when there is no radiation from the two primaries, but when one or two primaries radiate, the situation is changed.
In Figure 6(a), we plot the location of $L_{2}$ under the effect of changing $q_{1}$ (the radiation coefficient of the massive primary $1-\mu$ ) versus different mass ratios. The green, blue, orange, and red curves represent the effect of successive increase in radiation of the massive primary, respectively. As these curves illustrate, the greater the effect of the radiation (mass loss), the greater the negative nonlinear displacement 
of the location of $L_{2}$. As is observed at high radiation and greater mass ratios specifically at $\mu>0.038 L_{2}$ crosses to the left of less massive primary. When $\mu \in(0,0.2)$, the locations of $L_{2}$ have positive displacements, while this effect is reversed when $\mu \in(0.2,0.5)$. In Figure $6(\mathrm{~b})$ the situation is similar to that in Figure 6(b) but with different size of perturbations.

In Figure 6(c), we plot the location of $L_{2}$ under the effect of changing $q_{1}$ and $q_{2}$ (the radiation coefficient of the two primaries). The situation is a combination of the two effects depicted in Figures 6(a) and 6(b). Since the point $L_{2}$ is closer to less massive primary, the effect of the radiation of it is dominant.

Figures 6(d)-6(f) represent the location of Lagrangian point $L_{2}$ under the effect of changing $q_{1}$ and/or $q_{2}$ with relativistic corrections. In Figure $6(\mathrm{~d})$ we plot the location of $L_{2}$ under the effect of changing $q_{1}$ (the radiation coefficient of the less massive primary $\mu$ ) with relativistic corrections versus different mass ratios. The greater the effect of the radiation (mass loss), the greater the positive displacement of the location of $L_{2}$ in the domain $\mu \in(0,0.038)$. Beyond this domain, that is, when $\mu \in(0.038,0.5)$, the situation converses, but the nonlinearity becomes more visible. In Figure 6(e), the dynamics is similar to that in Figure 6(b) but it is clear with different size of perturbations. In Figure 6(f), we have combined effects of those depicted in Figures 6(d), and $6(\mathrm{e})$.

5.3. Analysis of $L_{3}$ Location. The black curve represents the case when the radiation pressure is totally neglected. In Figure 7(a) and in Figure 7(b), it seems that when $\mu=0.5$ the location of $L_{3}$ lies exactly at a normalized distance equal to 1.2 to the left of the barycenter, and when $\mu \rightarrow 0$ the location of $L_{3}$ lies exactly at a normalized distance equal to 1 to the left of the barycenter, and then the problem reduced to the classical two body problem. The same case also exists in Figure $7(\mathrm{c})$ when there is no radiation from the two primaries, but when one or two primaries radiate, the situation is changed.

In Figure $7(\mathrm{a})$, we plot the location of $L_{3}$ under the effect of changing $q_{1}$ (the radiation coefficient of the massive primary $1-\mu$ ) against different mass ratios. The green, blue, orange, and red curves represent the effect of successive increase in radiation of the massive primary, respectively. As these curves illustrate, the greater the effect of the radiation (mass loss) the greater the positive displacement of the location of $L_{3}$, that is, towards the massive primary.

In the case of sever radiation (the red curve) and mass ratios $\mu>0.15$ the order of the primaries RTBP may be reversed; for example, when $q_{1}>0.6$ the location of $L_{3}$ is weakly nonlinearly decreased. When $q_{1} \geq 0.8$ and $0.15<\mu<$ 0.35 the location of $L_{3}$ is exponentially decreased while when $0.35<\mu<0.5$ the location of $L_{3}$ is exponentially increased.

In Figure 7(b) we plot the location of $L_{3}$ under the effect of changing $q_{2}$ (the radiation coefficient of the less massive primary $\mu$ ) against different mass ratios. The greater the effect of the radiation (mass loss), the greater the positive displacement of the location of $L_{3}$, linearly during $q<0.4$ and nonlinearly during $q>0.4$. This effect becomes very clear for significantly large mass ratios. When comparing the two most left and the two most right parts on Figure 7(a) and Figure 7(b), they seemed in contrast to each other when $q<0.4$ due to the fact that the location of $L_{3}$ is closer to the massive primary than the less massive primary.

The analysis of Figure $7(d)$ is that the greater the effect of the radiation (mass loss), the greater the negative displacement of the location of $L_{3}$; that is to say, for $q<0.4$ the analysis of Figure $7(d)$ is in weak contrast to the analysis of Figure $7(\mathrm{a})$, while for $q>0.4$ and significantly large mass ratios we have again positive displacements.

The analyses of Figure 7(e) are in strong contrast to that of Figure 7(b) but with different sizes of perturbations due to the inclusion of the relativistic effects in addition to the photogravitational effects.

Figures 7(c)-7(f) represent the location of Lagrangian point $L_{3}$ under the effect of changing $q_{1}$ and $q_{2}$ without/with relativistic corrections, respectively. They represent the combined effects of those depicted in Figures 7(a) and 7(b) and in Figures $7(\mathrm{~d})$ and $7(\mathrm{e})$, respectively.

\section{Conclusion}

In a very important two works, Douskos and Perdios [7] and Ragos et al. [8] determined the stability of the equilibrium points and approximated the positions of the collinear points by series by expansions and determined the stability of the triangular as well as collinear points without taking into account the photo-gravitational effects of the two primaries. In our here presented work, we assumed the primaries to be radiant sources. The corrected locations of the collinear points are computed. Series forms of these location are obtained as new analytical results. In order to introduce a semianalytical view, A Mathematica program is constructed so as to draw the locations of collinear points versus the whole range of the mass ratio $\mu$ taking into account the photogravitational effects and/or the relativistic corrections. We analyzed all the obtained figures.

In a forthcoming works we aim to complete this work by performing the same computation to the triangular equilibrium points. Also we planned to study the stability of all equilibrium points; this would actually enhance the scientific significance of the current study.

\section{Appendices}

\section{A.}

The non-vanishing coefficients $\mathscr{M}_{k}^{L_{1}}$ and $\mathcal{N}_{k}^{L_{1}}$, of (19) are as follows:

$$
\begin{gathered}
\mathscr{M}_{0}^{L_{1}}=\frac{1-q_{1}}{1+8 q_{1}}, \\
\mathscr{M}_{1}^{L_{1}}=\frac{3 q_{1}}{\left(1+8 q_{1}\right)^{2}}\left(-1+28 q_{1}\right)\left(\frac{q_{2}}{q_{1}}\right)^{1 / 3}, \\
\mathscr{M}_{2}^{L_{1}}=\frac{q_{1}}{\left(1+8 q_{1}\right)^{3}}\left[-7+4\left(17-76 q_{1}\right) q_{1}\right]\left(\frac{q_{2}}{q_{1}}\right)^{2 / 3},
\end{gathered}
$$




$$
\begin{aligned}
& \mathscr{M}_{3}^{L_{1}}=\frac{1}{9\left(1+8 q_{1}\right)^{4}} \\
& \times\left[27\left(-1+q_{1}\right)\left(1+8 q_{1}\right)^{2}\right. \\
& \left.-\left(35+2 q_{1}\left(-777+8 q_{1}\left(213+292 q_{1}\right)\right)\right) q_{2}\right], \\
& \mathscr{M}_{4}^{L_{1}}=\frac{2}{9\left(1+8 q_{1}\right)^{5}} \\
& \times\left[27 q_{1}\left(1+8 q_{1}\right)^{2}\left(7+2 q_{1}\left(-19+56 q_{1}\right)\right)\right. \\
& +\left(-11+q_{1}\left(1013+8 q_{1}\right.\right. \\
& \left.\left.\times\left(-753+4 q_{1}\left(-317+884 q_{1}\right)\right)\right)\right) \\
& \left.\times q_{2}\right]\left(\frac{q_{2}}{q_{1}}\right)^{1 / 3} \\
& \mathcal{N}_{0}^{L_{1}}=\frac{1}{2\left(1+8 q_{1}\right)}\left(-1+q_{1}\right)\left(5+2 q_{1}\right), \\
& \mathcal{N}_{1}^{L_{1}}=\frac{3}{2\left(1+8 q_{1}\right)^{2}} \\
& \times\left[\left(1+q_{1}\left(31+6 q_{1}-56 q_{1}^{2}\right)\right)\right]\left(\frac{q_{2}}{q_{1}}\right)^{1 / 3}, \\
& \mathcal{N}_{2}^{L_{1}}=\frac{1}{\left(1+8 q_{1}\right)^{3}} \\
& \times\left[1+q_{1}\left(41+q_{1}\left(-153+172 q_{1}\left(-1+4 q_{1}\right)\right)\right)\right] \\
& \times\left(\frac{q_{2}}{q_{1}}\right)^{2 / 3} \\
& \mathcal{N}_{3}^{L_{1}}=-\frac{1}{18 q_{1}\left(1+8 q_{1}\right)^{4}} \\
& \times\left[27 q_{1}\left(1+8 q_{1}\right)^{2}\left(-5+q_{1}\left(3+4 q_{1}\left(1+4 q_{1}\right)\right)\right)\right. \\
& +2\left(15+q_{1}\left(367+q_{1}\right.\right. \\
& \times\left(11041+2 q_{1}\left(16125+8 q_{1}\right.\right. \\
& \left.\left.\left.\left.\left.\times\left(677+284 q_{1}\right)\right)\right)\right)\right) q_{2}\right], \\
& \mathcal{N}_{4}^{L_{1}}=\frac{1}{18 q_{1}\left(1+8 q_{1}\right)^{5}}\left(\frac{q_{2}}{q_{1}}\right)^{1 / 3} \\
& \times\left[27 q_{1}\left(1+8 q_{1}\right)^{2}\right. \\
& \times\left(5+2 q_{1}\left(-10+q_{1}\left(231+8 q_{1}\right.\right.\right. \\
& \left.\left.\left.\times\left(-29+104 q_{1}\right)\right)\right)\right) \\
& +2\left(4+q_{1}\left(222+q_{1}\left(-4381+2 q_{1}\right.\right.\right. \\
& \left.\left.\times\left(25441+8 q_{1}\left(20607+4 q_{1}\right)\right)\right)\right) \\
& \left.\left.\times\left(5671+1740 q_{1}\right)\right) q_{2}\right] \times\left(\frac{q_{2}}{q_{1}}\right)^{1 / 3},
\end{aligned}
$$$$
\begin{aligned}
\mathscr{N}_{5}^{L_{1}}= & -\frac{1}{54 q_{1}\left(1+8 q_{1}\right)^{6}} \\
& \times\left[2 7 q _ { 1 } ( 1 + 8 q _ { 1 } ) ^ { 2 } \left(9+2 q_{1}\right.\right. \\
& \times\left(319+4 q_{1}\left(25+q_{1}\right.\right. \\
& \left.\left.\left.\times\left(2523+8 q_{1}\left(-23+412 q_{1}\right)\right)\right)\right)\right) \\
& +2\left(-9+2 q_{1}\left(-228+q_{1}\right.\right. \\
& \times\left(7285+q_{1}\left(-184181+16 q_{1}\right.\right. \\
& \times\left(-62741+q_{1}\left(-34747+8 q_{1}\right.\right. \\
& \left.\left.\left.\left.\left.\left.\left.\times\left(10231+8644 q_{1}\right)\right)\right)\right)\right)\right)\right) q_{2}\right]\left(\frac{q_{2}}{q_{1}}\right)^{2 / 3}
\end{aligned}
$$

B.

The non-vanishing coefficients $\mathscr{M}_{k}^{L_{2}}$ and $\mathcal{N}_{k}^{L_{2}}$, of (26) are as follows:

$$
\begin{aligned}
& \mathscr{M}_{0}^{L_{2}}=\frac{-1+q_{1}}{1+8 q_{1}} \\
& \mathscr{M}_{1}^{L_{2}}=\frac{3 q_{1}}{\left(1+8 q_{1}\right)^{2}}\left(-1+28 q_{1}\right)\left(\frac{q_{2}}{q_{1}}\right)^{1 / 3} \\
& \mathscr{M}_{2}^{L_{2}}=\frac{q_{1}}{\left(1+8 q_{1}\right)^{3}} \\
& \times\left(7+4 q_{1}\left(-17+76 q_{1}\right)\right)\left(\frac{q_{2}}{q_{1}}\right)^{2 / 3}, \\
& \mathscr{M}_{3}^{L_{2}}=-\frac{1}{9\left(1+8 q_{1}\right)^{4}} \\
& \times\left[27\left(-1+q_{1}\right)\left(1+8 q_{1}\right)^{2}\right. \\
& +\left(35+2 q_{1}\left(-777+8 q_{1}\right.\right. \\
& \left.\left.\left.\times\left(213+292 q_{1}\right)\right)\right) q_{2}\right], \\
& \mathscr{M}_{4}^{L_{2}}=\frac{2}{9\left(1+8 q_{1}\right)^{5}} \\
& \times\left[27 q_{1}\left(1+8 q_{1}\right)^{2}\left(7+2 q_{1}\left(-19+56 q_{1}\right)\right)\right. \\
& +\left(11+q_{1}\left(-1013+8 q_{1}\right.\right. \\
& \times\left(753+4\left(317-884 q_{1}\right)\right. \\
& \left.\left.\left.\left.\times q_{1}\right)\right)\right) q_{2}\right]\left(\frac{q_{2}}{q_{1}}\right)^{1 / 3},
\end{aligned}
$$




$$
\begin{aligned}
&\left.\mathscr{M}_{5}^{L_{2}}=\frac{1}{9(1}+8 q_{1}\right)^{6} \\
& \times\left[9 q_{1}\left(1+8 q_{1}\right)^{2}\right. \\
& \times\left(-55+16 q_{1}\left(24+q_{1}\right.\right. \\
&\left.\left.\times\left(-279+304 q_{1}\right)\right)\right)+2\left(1+q_{1}\right. \\
& \times\left(1287+16 q_{1}\left(-778+q_{1}\right.\right. \\
&\left.\left.\left.\times\left(-397+8 q_{1}\left(1131+236 q_{1}\right)\right)\right)\right)\right) \\
&\left.\times q_{2}\right]\left(\frac{q_{2}}{q_{1}}\right)^{2 / 3}, \\
& \mathcal{N}_{0}^{L_{2}}= \frac{1}{2+16 q_{1}}\left[7+11 q_{1}\left(1+2 q_{1}\right)\right] \\
& \mathcal{N}_{1}^{L_{2}}= \frac{3}{2\left(1+8 q_{1}\right)^{2}} \\
& \times\left[1+q_{1}\left(25+2 q_{1}\right.\right. \\
&\left.\left.\times\left(7+12 q_{1}\right)\left(-3+16 q_{1}\right)\right)\right] \\
& \times\left(\frac{q_{2}}{q_{1}}\right)^{1 / 3}
\end{aligned}
$$

$$
\begin{aligned}
& \times\left.\left.\left.\left(-149+16 q_{1}\left(23+84 q_{1}\right)\right)\right)\right)\right) \\
&+ 2\left(7+q_{1}\left(343+4 q_{1}\left(-382+q_{1}\right.\right.\right. \\
& \times\left(17073+8 q_{1}\left(8977+8 q_{1}\right.\right. \\
& \times\left.\left.\left.\left.\left.\left.\left(2332+q_{1}\left(6521+9216 q_{1}\right)\right)\right)\right)\right)\right)\right) q_{2}\right], \\
& \mathcal{N}_{5}^{L_{2}}=\frac{1}{54 q_{1}\left(1+8 q_{1}\right)^{6}} \\
& \times\left[27 q_{1}\left(1+8 q_{1}\right)^{2}\right. \\
& \times\left(3+2 q_{1}\left(179+8 q_{1}\right.\right. \\
& \times\left(-278+q_{1}\left(-803+12 q_{1}\right.\right. \\
&\left.\left.\left.\left.\times\left(-949+12 q_{1}\left(-123+128 q_{1}\right)\right)\right)\right)\right)\right) \\
&+2\left(-9+2 q_{1}\left(-219+q_{1}\right.\right. \\
& \times\left(-15493+q_{1}\left(65081+16 q_{1}\right.\right. \\
& \quad \times\left(2888+q_{1}\left(-39569+16 q_{1}\right.\right. \\
& \quad \times\left(17815+2 q_{1}\right. \\
&\left.\left.\left.\left.\left.\left.\left.\quad \times\left(38069+54528 q_{1}\right)\right)\right)\right)\right)\right)\right)\right) \\
&\left.\quad \times q_{2}\right]\left(\frac{q_{2}}{q_{1}}\right)^{2 / 3} .
\end{aligned}
$$

$$
\begin{aligned}
\mathcal{N}_{2}^{L_{2}}= & \frac{1}{\left(1+8 q_{1}\right)^{3}} \\
\times & {\left[2+q_{1}\left(39+q_{1}\right.\right.} \\
& \times\left(847-48 q_{1}\left(-51+q_{1}\right.\right. \\
& \left.\left.\left.\left.\times\left(-89+64 q_{1}\right)\right)\right)\right)\right]\left(\frac{q_{2}}{q_{1}}\right)^{2 / 3},
\end{aligned}
$$$$
\mathcal{N}_{3}^{L_{2}}=\frac{1}{18 q_{1}\left(1+8 q_{1}\right)^{4}}
$$$$
\times\left[-27 q_{1}\left(1+8 q_{1}\right)^{2}\left(11+q_{1}\right.\right.
$$$$
\left.\times\left(67+4 q_{1}\left(59+44 q_{1}\right)\right)\right)
$$$$
+2\left(3+2 q_{1}\left(123+q_{1}\right.\right.
$$$$
\times\left(-957+q_{1}\left(15203+16 q_{1}\right.\right.
$$$$
\left.\left.\left.\left.\left.\times\left(4475+9926 q_{1}+9216 q_{1}^{2}\right)\right)\right)\right)\right) q_{2}\right],
$$

$$
\begin{aligned}
\mathcal{N}_{4}^{L_{2}}= & -\frac{1}{18 q_{1}\left(1+8 q_{1}\right)^{5}}\left(\frac{q_{2}}{q_{1}}\right)^{1 / 3} \\
& \times\left[2 7 q _ { 1 } ( 1 + 8 q _ { 1 } ) ^ { 2 } \left(-5+2 q_{1}\right.\right. \\
& \times\left(-14+q_{1}\left(-699+8 q_{1}\right.\right.
\end{aligned}
$$

C.

The non-vanishing coefficients $\mathscr{M}_{k}^{L_{3}}$ and $\mathscr{N}_{k}^{L_{3}}$ of (33) are as follows:

$$
\begin{aligned}
& \mathscr{M}_{0}^{L_{3}}=\frac{1}{3}\left(5+q_{1}\right), \\
& \mathscr{M}_{1}^{L_{3}}=\frac{1}{18}\left(-19-5 q_{1}+3 q_{2}\right), \\
& \mathscr{M}_{2}^{L_{3}}=\frac{7}{96 q_{2}^{2}}\left(-2 q_{1}^{2}\left(19+5 q_{1}\right)\right. \\
& +q_{1} q_{2}\left(73+23 q_{1}\right) \\
& \left.-4\left(7+5 q_{1}\right) q_{2}^{2}\right)\left(\frac{q_{2}}{q_{1}}\right)^{2}, \\
& \mathscr{M}_{3}^{L_{3}}=\frac{7}{2304 q_{2}^{3}} \\
& \times\left[-84 q_{1}^{3}\left(19+5 q_{1}\right)\right. \\
& +336 q_{1}^{2}\left(19+5 q_{1}\right) q_{2} \\
& -768 q_{1}\left(11+4 q_{1}\right) q_{2}^{2} \\
& \left.+\left(3248+1741 q_{1}\right) q_{2}^{3}\right] \\
& \times\left(\frac{q_{2}}{q_{1}}\right)^{3},
\end{aligned}
$$




$$
\begin{aligned}
& \mathscr{M}_{4}^{L_{3}}=\frac{7}{9216 q_{2}^{4}} \\
& \times\left[-588 q_{1}^{4}\left(19+5 q_{1}\right)\right. \\
& +414 q_{1}^{3}\left(19+5 q_{1}\right) q_{2} \\
& +9 q_{1}^{2}\left(5419+2173 q_{1}\right) q_{2}^{2} \\
& -q_{1}\left(84371+46834 q_{1}\right) q_{2}^{3} \\
& \left.+56\left(625+512 q_{1}\right) q_{2}^{4}\right] \\
& \times\left(\frac{q_{2}}{q_{1}}\right)^{4}, \\
& \mathscr{M}_{5}^{L_{3}}=\frac{49}{147465 q_{2}^{5}} \\
& \times\left[-2352 q_{1}^{5}\left(19+5 q_{1}\right)\right. \\
& -6096 q_{1}^{4}\left(19+5 q_{1}\right) q_{2} \\
& +12 q_{1}^{3}\left(59527+22817 q_{1}\right) q_{2}^{2} \\
& -12 q_{1}^{2}\left(96175+46304 q_{1}\right) q_{2}^{3} \\
& +q_{1}\left(795713+420985 q_{1}\right) q_{2}^{4} \\
& \left.-\left(214592+93715 q_{1}\right) q_{2}^{5}\right] \\
& \times\left(\frac{q_{2}}{q_{1}}\right)^{4} \\
& \mathcal{N}_{0}^{L_{3}}=\frac{1}{6}\left(5+q_{1}\left(-3+2 q_{1}\right)\right), \\
& \mathcal{N}_{1}^{L_{3}}=\frac{1}{72}\left[47-34 q_{1}^{2}+15 q_{2}\right. \\
& \left.-\frac{91 q_{2}}{q_{1}}+q_{1}\left(15+8 q_{2}\right)\right], \\
& \mathcal{N}_{2}^{L_{3}}=-\frac{1}{1728 q_{1}^{2}} \\
& \times\left[-2 q_{1}^{2}\left(-103+q_{1}\right.\right. \\
& \left.\times\left(537+50 q_{1}\right)\right)+q_{1} \\
& \times\left(1855+q_{1}\left(579+4882 q_{1}\right)\right) q_{2} \\
& \left.+6\left(-413+q_{1}\left(21+82 q_{1}\right)\right) q_{2}^{2}\right], \\
& \mathcal{N}_{3}^{L_{3}}=\frac{1}{124416 q_{1}^{3}} \\
& \times\left[12 q_{1}^{3}\left(1007+25 q_{1}\left(-57+14 q_{1}\right)\right)\right.
\end{aligned}
$$

$$
\begin{aligned}
& +72 q_{1}^{2}\left(2163+q_{1}\right. \\
& \left.\times\left(-1338+893 q_{1}\right)\right) q_{2}+84 q_{1} \\
& \times\left(-3040+q_{1} \times\left(2679+2615 q_{1}\right)\right) q_{2}^{2} \\
& -7\left(-15092+q_{1}\right. \\
& \left.\left.\times\left(18417+1594 q_{1}\right)\right) q_{2}^{3}\right], \\
& \mathcal{N}_{4}^{L_{3}}=\frac{1}{1492992 q_{1}^{4}} \\
& \times\left[8 4 q _ { 1 } ^ { 4 } \left(1007+25 q_{1}\right.\right. \\
& \left.\times\left(-57+14 q_{1}\right)\right) \\
& +6 q_{1}^{3}\left(357511+q_{1}\right. \\
& \left.\times\left(-27069+205210 q_{1}\right)\right) q_{2} \\
& -63 q_{1}^{2}\left(108347-7701 q_{1}\right. \\
& \left.+6582 q_{1}^{2}\right) q_{2}^{2}-7 q_{1} \\
& \times\left(-865669+2 q_{1}\right. \\
& \left.\times\left(8751+69208 q_{1}\right)\right) q_{2}^{3} \\
& +28\left(-53648+q_{1}\right. \\
& \left.\left.\times\left(-8295+5113 q_{1}\right)\right) q_{2}^{4}\right], \\
& \mathcal{N}_{5}^{L_{3}}=\frac{7}{71663616 q_{1}^{5}} \\
& \times\left[3 3 6 q _ { 1 } ^ { 5 } \left(1007+25 q_{1}\right.\right. \\
& \left.\times\left(-57+14 q_{1}\right)\right)+48 q_{1}^{4} \\
& \times\left(135337+q_{1}\right. \\
& \left.\times\left(29127+110680 q_{1}\right)\right) q_{2} \\
& -108 q_{1}^{3}\left(285685+3 q_{1}\right. \\
& \left.\times\left(21979+84386 q_{1}\right)\right) q_{2}^{2} \\
& +12 q_{1}^{2}\left(4088959+2 q_{1}\right. \\
& \left.\times\left(505899+2124101 q_{1}\right)\right) \\
& \times q_{2}^{3}-3 q_{1}\left(11569257+q_{1}\right. \\
& \left.\times\left(2427243+9605126 q_{1}\right)\right) q_{2}^{4} \\
& +7(1461824 \\
& \left.\left.+\left(122055-169714 q_{1}\right) q_{1}\right) q_{2}^{5}\right] .
\end{aligned}
$$




\section{Acknowledgments}

The authors are deeply indebted and thankful to the deanship of the scientific research and his helpful and distinct team of employees at Taibah University, Al-Madinah AlMunawwarah, Saudi Arabia. This research work was supported by Grant no. 3007/1434. The authors would also like to record their indebtedness and thankfulness to the reviewers for their valuable and fruitful comments as well as for their powerful reading and suggestions.

\section{References}

[1] S. Kumar and B. Ishwar, "Location of collinear equilibrium points in the generalized photogravitational elliptic restricted three body problem," International Journal of Engineering, Science and Technology, vol. 3, no. 2, pp. 157-162, 2011.

[2] A. Narayan and C. R. Kumar, "Stability of triangular equilibrium points in elliptical restricted three body problem under the effects of photogravitational and oblateness of primaries," International Journal of Pure and Applied Mathematics, vol. 70, no. 5, pp. 735-754, 2011.

[3] D. W. Schuerman, "The restricted three-body problem including radiation pressure," The Astrophysical Journal, vol. 238, no. 1, part 1, pp. 337-342, 1980.

[4] P. Oberti and A. Vienne, "An upgraded theory for Helene, Telesto, and Calypso," Astronomy and Astrophysics, vol. 397, no. 1, pp. 353-359, 2003.

[5] S. Kumar and B. Ishwar, "Solutions of generalized photogravitational elliptic restricted three body problem," in Proceedings of the International Conference on Modelling and Engineering and Technological Problems (ICMETP '09), vol. 1146 of AIP Conference Proceedings, pp. 456-461, January 2009.

[6] F. A. Abd El-Salam and S. Abd El-Bar, "Formulation of the postNewtonian equations of motion of the restricted three body problem," Applied Mathematics, vol. 2, no. 2, pp. 155-164, 2011.

[7] C. N. Douskos and E. A. Perdios, "On the stability of equilibrium points in the relativistic restricted three-body problem," Celestial Mechanics and Dynamical Astronomy, vol. 82, no. 4, pp. 317-321, 2002.

[8] O. Ragos, E. A. Perdios, V. S. Kalantonis, and M. N. Vrahatis, "On the equilibrium points of the relativistic restricted threebody problem," Nonlinear Analysis: Theory, Methods \& Applications, vol. 47, no. 5, pp. 3413-3418, 2001.

[9] M. P. Markakis, A. E. Perdiou, and C. N. Douskos, "The photogravitational Hill problem with oblateness: equilibrium points and Lyapunov families," Astrophysics and Space Science, vol. 315, no. 1-4, pp. 297-306, 2008.

[10] C. N. Douskos, “Collinear equilibrium points of Hill's problem with radiation and oblateness and their fractal basins of attraction," Astrophysics and Space Science, vol. 326, no. 2, pp. 263-271, 2010.

[11] R. K. Sharma, "The linear stability of libration points of the photogravitational restricted three-body problem when the smaller primary is an oblate spheroid," Astrophysics and Space Science, vol. 135, no. 2, pp. 271-281, 1987.

[12] P. P. Hallan and N. Rana, "The existence and stability of equilibrium points in the Robe's restricted three-body problem," Celestial Mechanics and Dynamical Astronomy, vol. 79, no. 2, pp. 145-155, 2001.
[13] Z. Sándor and B. Érdi, "Symplectic mapping for Trojan-type motion in the elliptic restricted three-body problem," Celestial Mechanics \& Dynamical Astronomy, vol. 86, no. 4, pp. 301-319, 2003.

[14] L. Floría, "On an analytical solution in the planar elliptic restricted three-body problem," in VIII Journées Zaragoza-Pau de Mathématiques Appliquées et de Statistiques, vol. 31, pp. 135144, Prensas Universitarias de Zaragoza, Zaragoza, Spain, 2004.

[15] A. P. Markeev, "On one special case of parametric resonance in problems of celestial mechanics," Astronomy Letters, vol. 31, no. 5, pp. 350-356, 2005.

[16] J. F. Palacián, P. Yanguas, S. Fernández, and M. A. Nicotra, "Searching for periodic orbits of the spatial elliptic restricted three-body problem by double averaging," Physica D, vol. 213, no. 1, pp. 15-24, 2006.

[17] J. F. Palacián and P. Yanguas, "From the circular to the spatial elliptic restricted three-body problem," Celestial Mechanics and Dynamical Astronomy, vol. 95, no. 1-4, pp. 81-99, 2006.

[18] A. D. Bruno and V. P. Varin, "On families of periodic solutions of the restricted three-body problem," Celestial Mechanics and Dynamical Astronomy, vol. 95, no. 1-4, pp. 27-54, 2006.

[19] M. K. Ammar, "The effect of solar radiation pressure on the Lagrangian points in the elliptic restricted three-body problem," Astrophysics and Space Science, vol. 313, no. 4, pp. 393-408, 2008.

[20] A. Narayan and C. Ramesh, "Stability of triangular points in the generalised oblate elliptical restricted three body problem," Modelling, Measurement and Control B, vol. 77, no. 1-2, pp. 1-19, 2008.

[21] A. Narayan and C. Ramesh, "Resonance stability of triangular equilibrium points of the planar elliptical restricted three-body problem in which both of the primaries are oblate spheroid," Modelling, Measurement and Control B, vol. 77, no. 5-6, pp. 5573, 2008.

[22] A. Narayan and C. Ramesh Kumar, "Parametric resonance stability of triangular equilibrium points of the planar elliptical restricted three-body problem around the primaries of Oblate Spheroid," Modelling, Measurement and Control B, vol. 78, no. 3-4, pp. 1-20, 2009.

[23] B. S. Kushvah, "Linear stability of equilibrium points in the generalized photogravitational Chermnykh's problem," Astrophysics and Space Science, vol. 318, no. 1-2, pp. 41-50, 2008.

[24] B. S. Kushvah, "The effect of radiation pressure on the equilibrium points in the generalized photogravitational restricted three body problem," Astrophysics and Space Science, vol. 315, no. 1-4, pp. 231-241, 2008.

[25] B. S. Kushvah, "Trajectories of L4 and Lyapunov characteristic exponents in the generalized photogravitational Chermnykhlike problem," Astrophysics and Space Science, vol. 333, no. 1, pp. 49-59, 2011.

[26] C. N. Douskos, "Equilibrium points of the restricted three-body problem with equal prolate and radiating primaries, and their stability," Astrophysics and Space Science, vol. 333, no. 1, pp. 7987, 2011.

[27] J. Shankaran, P. Sharma, and B. Ishwar, "Equilibrium points in the generalized photogravitational non-planar restricted three body problem," International Journal of Engineering, Science and Technology, vol. 3, no. 2, pp. 63-67, 2011.

[28] V. A. Brumberg, Relativistic Celestial Mechanics, Nauka, Moscow, Russia, 1972.

[29] K. B. Bhatnagar and P. P. Hallan, "Existence and stability of $\mathrm{L}_{4,5}$ in the relativistic restricted three-body problem," Celestial 
Mechanics and Dynamical Astronomy, vol. 69, no. 3, pp. 271-281, 1997.

[30] R. K. Sharma, W. Fricke, and G. Teleki, Eds., Sun and Planetary System, D. Reidel Publishing Company, Dordrecht, The Netherlands, 1982.

[31] J. F. L. Simmons, A. J. C. McDonald, and J. C. Brown, "The restricted 3-body problem with radiation pressure," Celestial Mechanics, vol. 35, no. 2, pp. 145-187, 1985.

[32] Yu. A. Chernikov, "The photogravitational restricted threebody problem," Soviet Astronomy, vol. 14, no. 1, pp. 176-181, 1970.

[33] M. H. Soffel, Relativity in Astrometry, Celestial Mechanics and Geodesy, Springer, Berlin, Germany, 1989. 


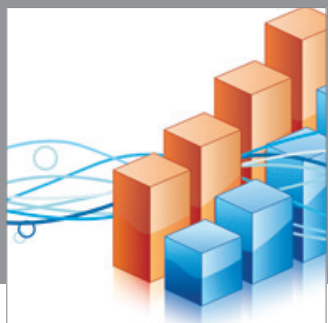

Advances in

Operations Research

mansans

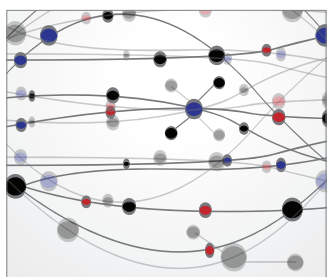

The Scientific World Journal
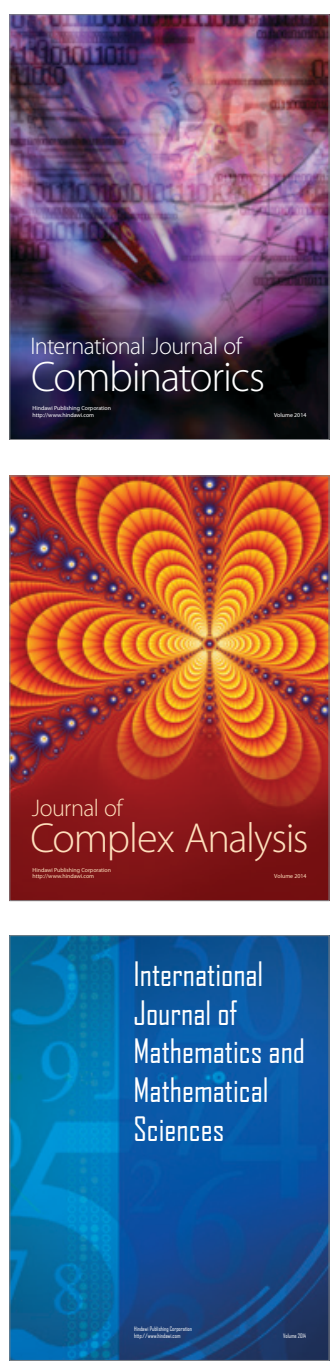
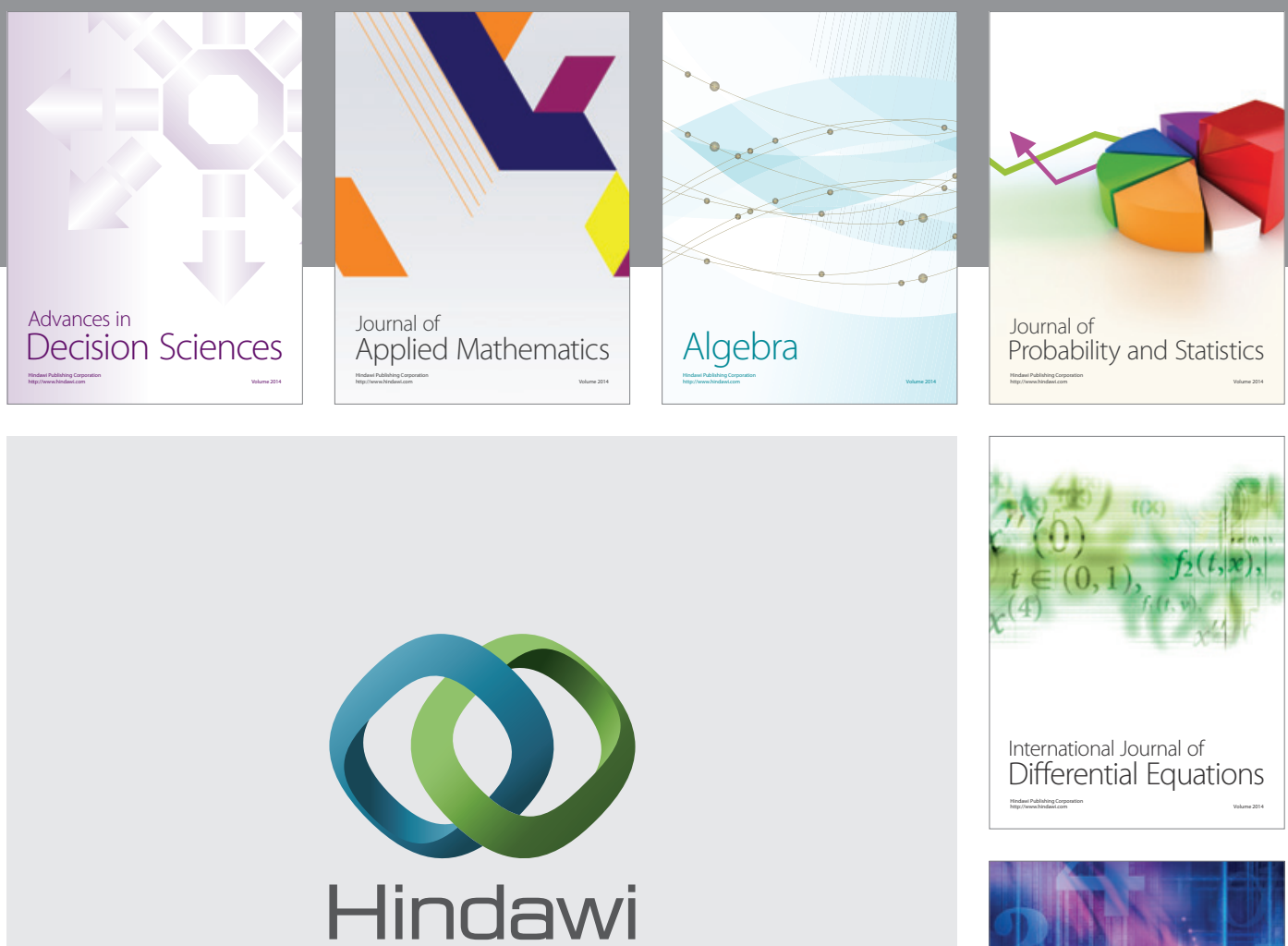

Submit your manuscripts at http://www.hindawi.com
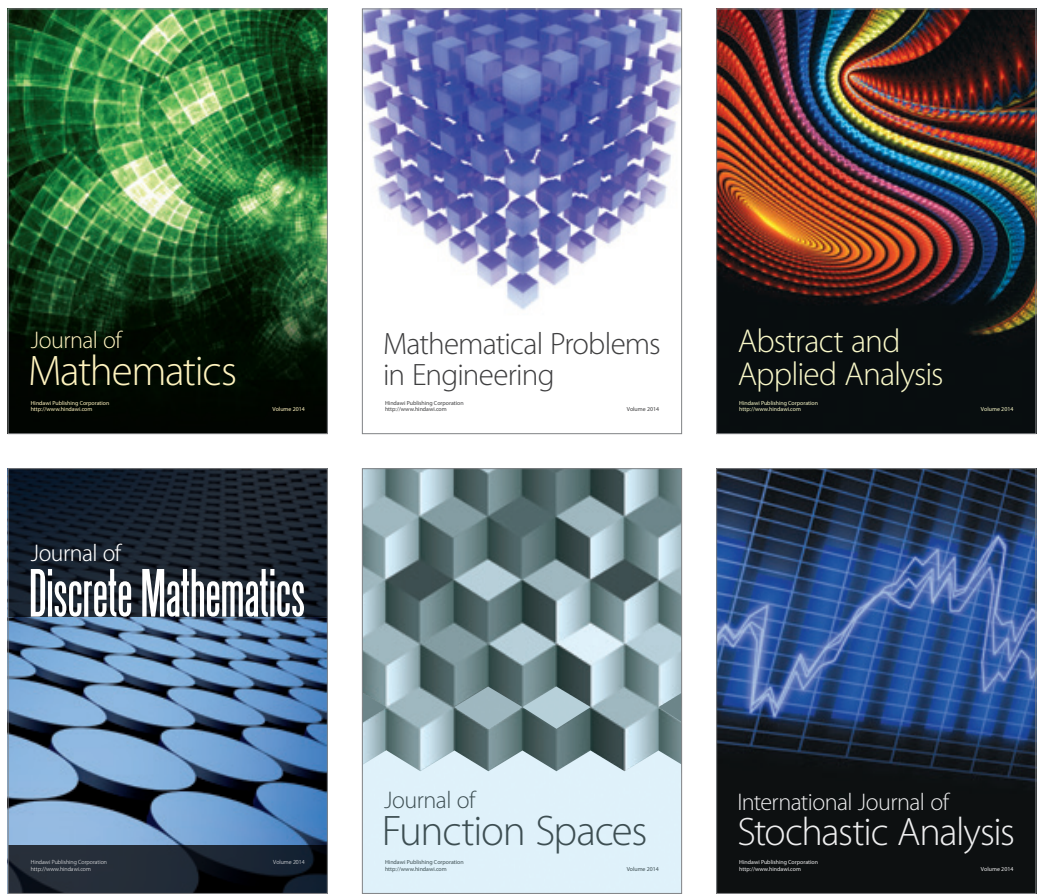

Journal of

Function Spaces

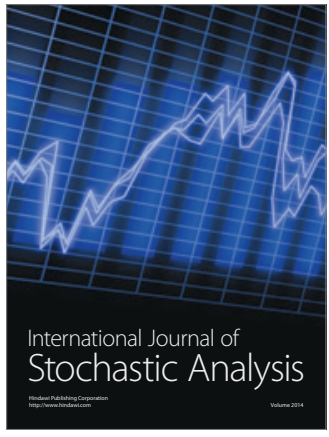

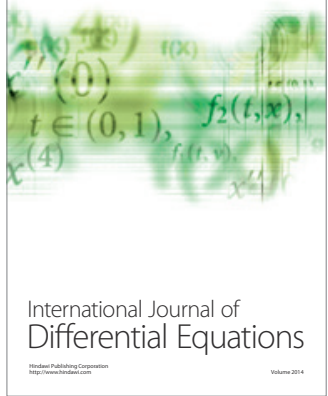
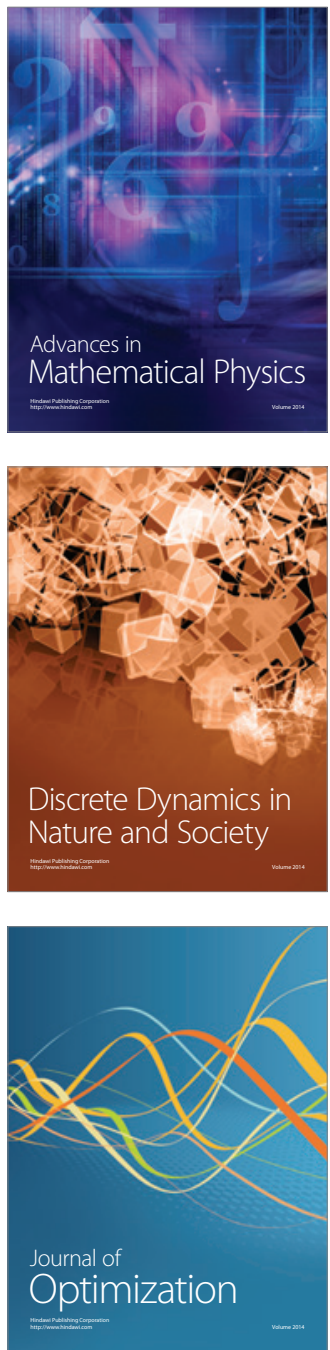